\title{
The phospho-occupancy of an atypical SLIMB-binding site on PERIOD that is phosphorylated by DOUBLETIME controls the pace of the clock
}

\author{
Joanna C. Chiu, ${ }^{1}$ Jens T. Vanselow, ${ }^{2}$ Achim Kramer, ${ }^{2}$ and Isaac Edery ${ }^{3,4}$ \\ ${ }^{1}$ Rutgers University, Center for Advanced Biotechnology and Medicine, Piscataway, New Jersey 08854, USA; ${ }^{2}$ Laboratory \\ of Chronobiology, Charité Universitätsmedizin Berlin, 10115 Berlin, Germany; ${ }^{3}$ Department of Molecular Biology \\ and Biochemistry, Rutgers University, Center for Advanced Biotechnology and Medicine, Piscataway, New Jersey 08854, \\ USA
}

A common feature of animal circadian clocks is the progressive phosphorylation of PERIOD (PER) proteins, which is highly dependent on casein kinase $\mathrm{I} \delta / \varepsilon(\mathrm{CKI} \delta / \varepsilon$; termed DOUBLETIME [DBT] in Drosophila) and ultimately leads to the rapid degradation of hyperphosphorylated isoforms via a mechanism involving the F-box protein, $\beta$-TrCP (SLIMB in Drosophila). Here we use the Drosophila melanogaster model system, and show that a key step in controlling the speed of the clock is phosphorylation of an N-terminal Ser (S47) by DBT, which collaborates with other nearby phosphorylated residues to generate a high-affinity atypical SLIMB-binding site on PER. DBT-dependent increases in the phospho-occupancy of S47 are temporally gated, dependent on the centrally located DBT docking site on PER and partially counterbalanced by protein phosphatase activity. We propose that the gradual DBT-mediated phosphorylation of a nonconsensus SLIMB-binding site establishes a temporal threshold for when in a daily cycle the majority of PER proteins are tagged for rapid degradation. Surprisingly, most of the hyperphosphorylation is unrelated to direct effects on PER stability. We also use mass spectrometry to map phosphorylation sites on PER, leading to the identification of a number of "phospho-clusters" that explain several of the classic per mutants.

[Keywords: Circadian rhythms; Drosophila; PER; $\beta$-TrCP/SLIMB; CK1ع/DBT; F-box protein; phosphorylation]

Supplemental material is available at http://www.genesdev.org.

Received April 9, 2008; revised version accepted May 9, 2008.

Circadian $(\cong 24 \mathrm{~h})$ rhythms are widespread in all kingdoms of life and are driven by cellular "clocks" or pacemakers (for review, see Roenneberg and Merrow 2005). These pacemakers are based on the expression of a core set of species or tissue specific "clock" genes that are central to rhythm generation. Much of the earlier work led to the realization that a major feature of intracellular clock mechanisms involves interconnected positive and negative translational-transcriptional feedback loops that yield cyclical gene expression in one or more of the core clock genes (Dunlap 1999).

Despite the overwhelming success of this molecular framework featuring rhythmic gene expression, work in the last several years has demonstrated the importance of post-transcriptional regulation, especially protein phosphorylation, in generating cellular oscillators with circadian properties (Bae and Edery 2006; Merrow et al. 2006;

${ }^{4}$ Corresponding author.

E-MAIL edery@cabm.rutgers.edu; FAX (732) 235-5318.

Article is online at http://www.genesdev.org/cgi/doi/10.1101/gad.1682708.
Gallego and Virshup 2007). Most notably, it appears that a shared feature of all circadian systems analyzed to date is that a major protein component functioning within the negative limb of circadian transcription undergoes daily cycles in phosphorylation that are central to clock progression. This was first observed for the Drosophila melanogaster PERIOD (dPER) protein (Edery et al. 1994). Besides dPER, major components of the clock in this system include TIMELESS (TIM), CLOCK (dCLK), and CYCLE (CYC) (Hardin 2005). dCLK and CYC are transcription factors of the basic helix-loop-helix (bHLH)/ PAS (Per-Arnt-Sim) superfamily that heterodimerize to stimulate the daily transcription of $d p e r$ and tim, in addition to other clock and downstream genes.

Phosphorylation has been linked to regulation of dPER stability, nucleocytoplasmic distribution, and transcriptional repressor potency (Bae and Edery 2006). Newly synthesized dPER is initially present as a hypophosphorylated variant(s) in the late day/early night, progressively increasing in extent of phosphorylation such that by the late night/early day only hyperphosphorylated species 
are detected (Edery et al. 1994). The DOUBLETIME (DBT) kinase (homolog of mammalian CKI $\delta / \varepsilon$ ) is a key kinase controlling the temporal program underlying dPER phosphorylation and stability (Kloss et al. 1998; Price et al. 1998). As the levels of TIM increase during the day it binds dPER and somehow protects it against DBT-mediated degradation (Price et al. 1995; Rothenfluh et al. 2000; Ko et al. 2002). The interaction of dPER with TIM also stimulates but is not obligatory for nuclear localization of both proteins (Shafer et al. 2002; Meyer et al. 2006). In the nucleus, dPER binds to dCLK and blocks its trans-activation potential (Darlington et al. 1998; Lee et al. 1998, 1999). During the late-night/early morning declining levels of nuclear TIM somehow accelerate hyperphosphorylation of dPER, whereby highly phosphorylated isoforms are targeted to the $26 \mathrm{~S}$ proteasome by the F-box protein SLIMB (Drosophila homolog of $\beta$-TrCP) (Grima et al. 2002; Ko et al. 2002). In general, F-box proteins recognize phosphorylated substrates and are part of larger SCF (Skp1, Cullin, F-box) E3 ligases that target substrates to the ubiquitin-proteasome pathway (Ho et al. 2008). Rapidly declining levels of dPER in the nucleus severely diminish or terminate its repressor function, initiating another round of dCLK-CYC-mediated transcription. Thus, a key aspect of the temporal regulation in dCLK-CYC activity involves the phase-specific accumulation and duration of dPER in the nucleus.

The progressive phosphorylation of dPER throughout a daily cycle and its ability to act as a transcriptional repressor are dependent on a small DBT-binding domain (dPER DBT-binding domain; dPDBD) in the central region of dPER (Kim et al. 2007; Nawathean et al. 2007). In the absence of the dPDBD, dPER is very stable, mainly hypophosphorylated, and has weak ability to inhibit dCLK, suggesting that the phosphorylated state of dPER also modulates its potency as a transcriptional repressor (Nawathean and Rosbash 2004; Kim et al. 2007; Nawathean et al. 2007). CKII and possibly other kinases also regulate dPER levels, although they appear to play less significant roles compared with DBT and might contribute in secondary pathways such as via regulating subcellular distribution. In addition to kinases, protein phosphatase 1 (PP1) and 2A (PP2A) regulate the phosphorylated state of dPER and other clock proteins, presumably antagonizing the destabilizing effects of phosphorylation on clock protein stability (Sathyanarayanan et al. 2004; Kim and Edery 2006; Fang et al. 2007).

A strikingly similar scenario also occurs in mammals, whereby CKI $\delta / \varepsilon$ plays a major role in regulating daily cycles in the phosphorylation and abundance of mammalian PERs (mPER1-3) (Gallego and Virshup 2007). The importance of PER phosphorylation is highlighted by studies showing that mutations in either a phosphorylation site on human PER2 or CKIס underlie several familial advanced sleep phase syndromes (FASPS) (Toh et al. 2001; Xu et al. 2005, 2007; Vanselow et al. 2006). Despite much progress, a detailed understanding of of the phosphorylation events and the role of $\mathrm{CKI} \varepsilon / \mathrm{DBT}$ in the phase-specific degradation of PER proteins is lacking. Herein, we use the $D$. melanogaster system to deter- mine how phosphorylation regulates the daily decline in PER levels. Our results indicate that gradual DBT-mediated increases in the phospho-occupancy of Ser47 on dPER acts like a "throttle" by timing when in the day the majority of dPER proteins are tagged for efficient recognition by SLIMB, a critical event in setting the pace of the clock. In addition, it appears that much, if not all of the more centrally localized phosphorylation on dPER is not directly involved in regulating stability, suggesting that the majority of the hyperphosphorylation observed for PER proteins is involved in other biochemical functions. Finally, using mass spectrometry we show that many of the phosphorylation sites on dPER are organized in local "clusters" and that the classic per ${ }^{\mathrm{S}(=\text { Short })}$ mutation (Konopka and Benzer 1971) abolishes a phosphorylation site.

\section{Results}

\section{Ser47 is a key determinant regulating dPER stability}

We previously showed that the DBT-dependent progressive phosphorylation and subsequent SLIMB-mediated degradation of dPER can be recapitulated in cultured Drosophila Schneider (S2) cells by expressing recombinant $d p e r$ and $d b t$, whereby expression of $d b t$ is controlled by the copper-inducible metallothionein promoter (pMT) and that of dper by the constitutive actin5C promoter (pAct) (Ko et al. 2002). To identify regions regulating dPER stability we analyzed a range of deletion mutants using this system. In one such series, we generated five V5-tagged dPER mutants, each containing nonoverlapping 100-amino-acid deletions that span the first 500 amino acid residues. Although all five dPER deletion mutants exhibit $d b t$-induced increases in phosphorylation (indicated by temporal decreases in electrophoretic mobility) that are similar to those observed for wild-type dPER, only $\operatorname{dPER}(\Delta 2-100)$ proteins remained stable (Fig. 1A, lanes 5-8; data not shown; in the experiment shown, dPER levels exhibit a noticeable decline at $12 \mathrm{~h}$ post- $d b t$ induction, whereas in most experiments this occurs later). Our results not only indicate that amino acids 2-100 are critical for DBT-mediated degradation but also demonstrate that global hyperphosphorylation per se is not sufficient to trigger the rapid proteolysis of dPER.

One possibility explaining the enhanced stability of $\operatorname{dPER}(\Delta 2-100)$ proteins is that amino acids $2-100$ contain sequence elements that mediate the interaction of dPER with SLIMB. Indeed, amino acids 17-23 and amino acids 47-55 show loose similarity to the standard SLIMB/ $\beta$-TrCP consensus recognition motif of DpSGФX ${ }_{1+n} \mathrm{pS}$ (pS, phosphorylated Ser; $\Phi$, any hydrophobic amino acid; $\mathrm{X}$, any amino acid) (Supplemental Fig. S1; e.g., Fuchs et al. 2004). A series of smaller deletions targeting these regions focused our attention on amino acids 44-51 (Supplemental Fig. S2A). This region contains four serine residues-S44, S45, S47, and S48-that are highly conserved in per genes from insects, raising the possibility that one or more of these is a phospho-determinant in regulating dPER stability. Indeed, a dPER variant in which 


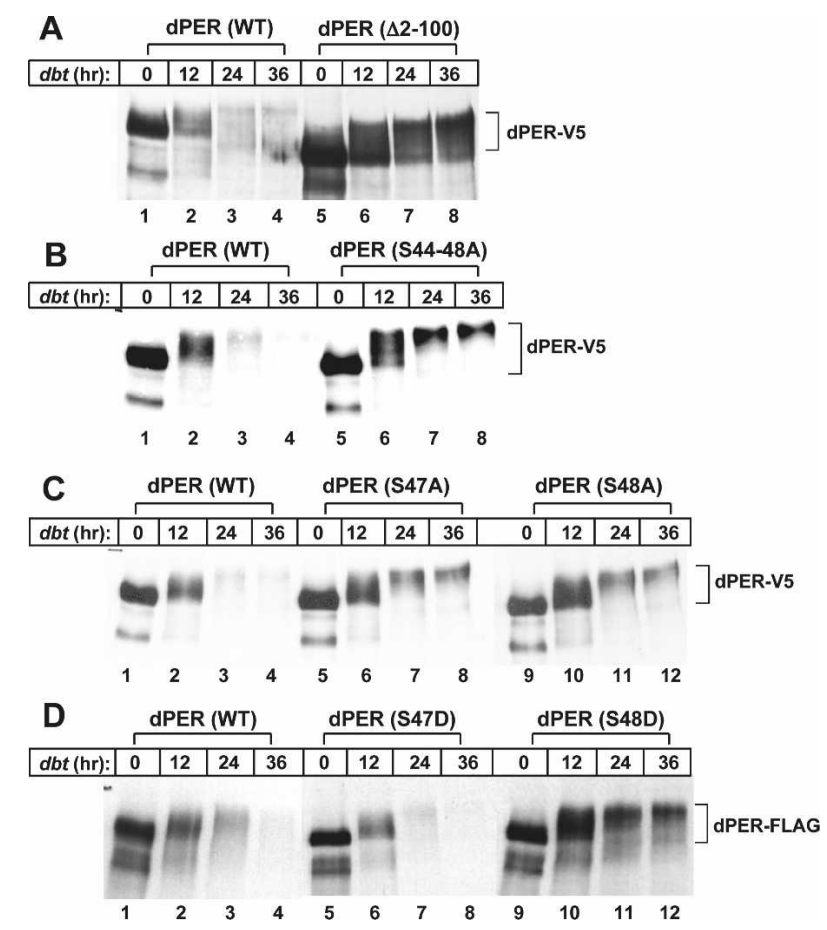

Figure 1. Identification of $\mathrm{N}$-terminal sequences required for DBT-mediated degradation of dPER in cultured S2 cells. S2 cells were cotransfected with wild-type or mutant variants of $d p e r$ containing plasmids (pAc-dper-V5-His $[A-C]$ or pAc-3XFlagHis-dper/Tev100-6Xc-myc [D]) and pMT- $d b t$-V5-His, and collected at the indicated times (hours) post- $d b t$ induction. dPER variants were detected by immunoblotting in the presence of $\alpha$-V5 $(A-C)$ or $\alpha$-Flag antibodies $(D)$.

all four Ser were substituted with Ala, dPER(S44-48A), phenocopied the enhanced stability of dPER( $\Delta 44-51)$ (cf. Fig. 1B and Supplemental Fig. S2A).

Single Ser-to-Ala mutants indicate that S47 and S48 play a major role in DBT-mediated dPER turnover (Fig. 1C; Supplemental Fig. S2B). In the case of S44 and S45, we noted a small but reproducible increase in dPER stability for the double mutant (S44/45A) but not when singly evaluated (Supplemental Fig. S2B; data not shown), suggesting minor roles. Replacing S47 with Asp (S47D) to mimic phosphorylation accelerates the kinetics of dPER disappearance following induction of $d b t$ (Fig. 1D). However, the dPER(S48D) variant is stable (Fig. 1D), suggesting that S48 modulates dPER stability in a manner independent of phosphorylation at this site. The increased stability of the different dPER mutants we analyzed were confirmed by measuring degradation rates after $d b t$ induction by treating cells with cycloheximide to block de novo protein synthesis (data not shown). Although S47 is a key DBT-dependent phospho-determinant in regulating dPER stability, other regions within the first 100 amino acids make contributions. Together, the results strongly suggest the following ranking, beginning with the most stable dPER variants; $\operatorname{dPER}(\Delta 2-$ 100) > dPER $(\Delta 44-51), \quad$ dPER $($ S44-48A) $>$ dPER $(S 47 / 48 \mathrm{~A})>$ dPER $($ S47A $)>$ dPER $($ S44/45A $)>$ dPER $(W T)$.

\section{Using a TEV/TAG strategy to examine phosphorylation in the first 100 amino acids of dPER}

To enhance the detection of phosphorylation-dependent electrophoretic mobility changes in the first 100 amino acids of dPER we devised a strategy, which we call TEV/ TAG (Fig. 2A), whereby we expressed a dPER version with a TEV protease site inserted at amino acid 100 (dPER/TEV100). To visualize the amino acid 1-100 fragment after TEV cleavage, we used $16 \%$ Tris-glycine gels and blotted with $\alpha$-Flag antibodies, whereas $6 \%$ gels and $\alpha$-c-myc antibodies were used to monitor the larger amino acid 101-1224 fragment. We placed TEV/TAG sites in several different positions on dPER and other test proteins and routinely obtain near total cleavage at the desired site (e.g., Fig. 3; data not shown). The location for TEV site insertion is chosen based on the prediction by protein secondary structure analysis, suggesting a low probability of disrupting important protein structural elements and high probability of access by the TEV protease (data not shown). For dPER, we ensured that any TEV/TAG derivative meets certain functional criteria, like DBT-dependent hyperphosphorylation, ability to inhibit dCLK-dependent transcription, and interaction with TIM (Fig. 2; data not shown).

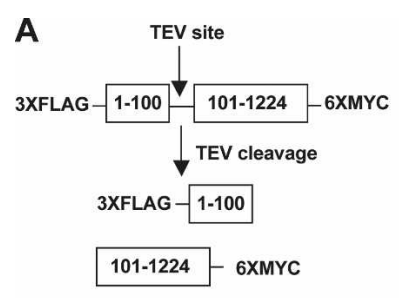

C

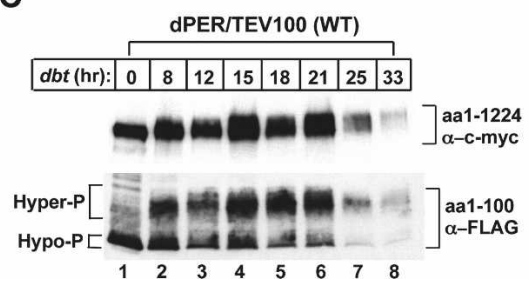

Figure 2. Using the TEV/TAG strategy to evaluate phosphorylation in the first 100 amino acids of dPER. (A) Schematic model showing the pAc-3XFlag-His-dper/Tev100-6Xc-myc construct and resulting cleavage products. $(B)$ Extracts were prepared from S2 cells expressing pAc-3XFlag-His-dper/Tev100$6 \mathrm{Xc}-\mathrm{myc}$ with $(+)$ or without $(-)$ coexpression of pMT-dbt-V5His. (Right) Extracts were subjected to TEV cleavage, incubated with $\alpha$-Flag beads, and immune complexes treated with $\lambda$-phosphatase $(+)$ or mock treated $(-)$. dPER(1-100) was detected using $\alpha$-Flag antibodies. Hypo- and hyperphosphorylated isoforms are indicated. $\left(^{\star}\right)$ Hyperphosphorylated isoforms only detected in the presence of induced $d b t$. (C) S2 cells coexpressing pAc3XFlag-His-dper/Tev100-6Xc-myc and pMT-dbt-V5-His were harvested at the indicated times post- $d b t$ induction and extracts were either TEV-treated or mock-treated. Mock-treated extracts were subjected to immunoblotting in the presence of $\alpha$-c-myc antibodies to detect full-length dPER (top), and TEV-treated extracts were used to detect $\mathrm{dPER}(1-100)$ using $\alpha$-Flag antibodies (bottom). 
A

A

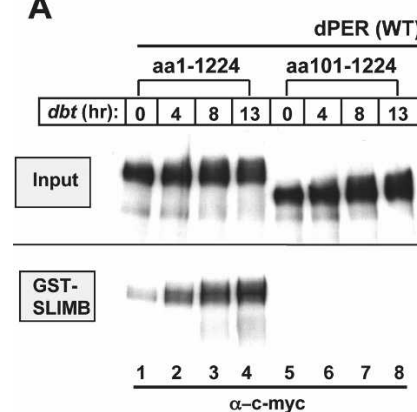

C

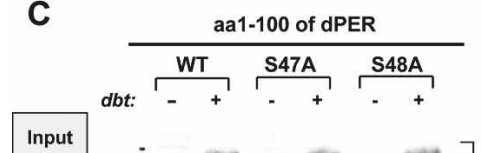

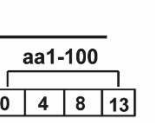

B

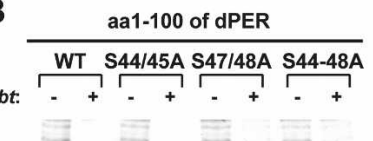

Figure 3. S47 is a key phospho-determinant mediating dPER-SLIMB interactions. $(A)$ S2 cells coexpressing pAc-3XFlag-His-dper/ Tev100-6Xc-myc and pMT-dbt-V5-His were collected at the indicated times post- $d b t$ induction. Extracts were either treated with TEV to yield amino acids 1-100 or amino acids 101-1224, or mock-treated retaining the full-length amino acids 1-1224. The top panel shows the input used in GST-SLIMB pulldown assays and the bottom panel shows the bound dPER proteins. The amino acid 1-100 fragment was detected using $\alpha$-Flag antibodies, whereas full-length dPER and the amino acid 101-1224 fragment were detected using $\alpha$-c-myc antibodies. Hyperphosphorylated and hypophosphorylated dPER isoforms are indicated as Hyper-P and Hypo-P, respectively. $(B-D)$ Extracts collected from S2 cells expressing wild-type (WT) or mutant derivatives of pAc-3XFlag-His-dper/Tev100-6Xcmyc with $(+)$ or without $(-)$ induced $d b t$ were subjected to TEV cleavage, subjected to GSTSLIMB pull-down assays and the amino acid 1-100 dPER fragment detected with $\alpha$-Flag antibodies. $(B)$ Asterisk $\left({ }^{\star}\right)$ denotes S44-48A mutant hyperphosphorylated isoforms with faster mobility as compared with wild-type hyperphosphorylated isoforms.
Using the TEV/TAG strategy, we determined that in our S2 cell system there is some phosphorylation within the first 100 amino acids of dPER even in the absence of induced DBT, although the majority is hypo- or nonphosphorylated (Fig. 2B, lanes 1,2). The induction of DBT greatly stimulates the "conversion" of non/hypo- to hyperphosphorylated isoforms (Fig. 2B, lanes 1,3), and in addition leads to the production of novel more highly phosphorylated isoforms (Fig. 2B, lane 3, asterisk). Phosphatase $(\lambda \mathrm{PP})$ treatment confirmed that the observed slower migrating $\alpha$-Flag immunoreactive bands are due to phosphorylation (Fig. 2B, lanes 2,4). The presence of several mobility variants (e.g., Fig. 2B, lane 3) strongly suggests that multiple residues are phosphorylated (see Table 2, below). Following induction of $d b t$ the ratio of highly phosphorylated isoforms to the non/hypophosphorylated version progressively increases, peaking $\sim 18$ $\mathrm{h}$ post- $d b t$ induction (Fig. 2C, cf. lanes 2 and 5). We did not observe the accumulation of slower migrating isoforms of the 1-100-amino-acid fragment with longer $d b t$ induction periods or in the presence of proteasome inhibitors, suggesting that the hyperphosphorylated species detected are maximally phosphorylated (data not shown). As expected, decreases in the levels of the dPER fragment containing the first 100 amino acids coincide with the degradation kinetics of the full-length protein, with substantial decreases by $25 \mathrm{~h}$ post- $d b t$ induction (Fig. 2C, top panel, cf. lanes 2 and 7). Our results indicate that the DBT-dependent transition in the first 100 amino acids of dPER from mainly hypophosphorylated to largely maximally phosphorylated is gradual and that the rapid decline in dPER levels occurs several hours after a substantial fraction of the molecules attain a highly phosphorylated state in the first 100 amino acids.

\section{Phosphorylation of S47 is critical for SLIMB binding to $d P E R$}

We combined the TEV/TAG strategy with SLIMB fused to glutathione-S-transferase (GST) to identify the SLIMBbinding region on APER. The results clearly indicate that amino acids 1-100 of dPER are sufficient and necessary for binding to SLIMB (Fig. 3A). No binding was observed with a GST-control resin (data not shown). Binding of the 1-100 amino acid dPER fragment to SLIMB is strongly enhanced by DBT-induced phosphorylation (Fig. 3A, lanes 9-12). The low level of dPER-SLIMB interaction detected at time 0 before $d b t$ induction can possibly be attributed to endogenous $d b t$ expression or the leakiness of the pMT promoter that drives the $d b t$ transgene (Fig. 3A, lanes 1,9). Although the 101-1224 amino acid fragment is responsible for much of the phosphorylation-mediated mobility shifts in dPER (e.g., Fig. 1A) and contains the DBT docking site (Kim et al. 2007; Nawathean et al. 2007), it showed no detectable binding to SLIMB (Fig. 3A, lanes 5-8), indicating that the extensive global hyperphosphorylation of dPER is not sufficient for SLIMB binding. Rather, there is a clear link between DBT-mediated phosphorylation in the first 100 amino acids and the efficiency of dPER binding to SLIMB (Fig. 3A, lanes 9-12).

Mutations in the first 100 amino acids that stabilize dPER (e.g., S44-48A, S47A, S48A, and S48D) strongly inhibit binding to SLIMB (Fig. 3B-D). Importantly, the 
S47D mutation, which renders the full-length protein less stable (Fig. 1D), has a much higher affinity for SLIMB (Fig. 3D, lanes 1-4; cf. input levels to staining intensities of bound material). For example, in the absence of induced DBT the "hypophosphorylated" first 100 amino acids of the dPER(S47D) variant exhibits strong binding to SLIMB, unlike the wild-type version (Fig. 3D, lanes 1,3). Nonetheless, the binding of the amino acid 1-100 fragment from dPER(S47D) is further stimulated by exogenously expressed DBT (Fig. 3D, lanes 3,4). Thus, although S47 is likely to be the key phosphodeterminant in mediating dPER-SLIMB interactions, DBT-dependent phosphorylation of other Ser/Thr residues in the first 100 amino acids of dPER also contribute. For example, the double mutant S44/45A partially reduces binding to SLIMB (Fig. 3B, lanes 2,4), consistent with its smaller effect on dPER levels (Supplemental Fig. S2B, lanes 3,7). That other residues in the first 100 amino acids of dPER are phosphorylated besides those between amino acid 44 and amino acid 48 is evident from the DBT-dependent shift in the mobility of the dPER(S44-48A) fragment (Fig. 3B, lane 8), albeit resulting in isoforms with faster mobilities, almost certainly due to blocking phosphorylation of Ser residues within amino acids 44-48 (see Table 2, below). However, unlike dPER(S44-48A) we did not observe noticeable alterations in the mobilities of the first 100amino-acid fragment derived from the $\operatorname{dPER}(\mathrm{S} 47 \mathrm{~A})$ and dPER(S48A) versions following $d b t$ induction (Fig. 3C), indicating that under the conditions used, single changes in the phosphorylated status of S47 do not yield noticeable electrophoretic mobility differences (although the status of S47 phosphorylation could affect mobility in combination with other mutations). We obtained similar results with regards to mutant effects on the efficiency of SLIMB binding when evaluating full-length versions of dPER in the absence of TEV cleavage (data not shown).

Together, the results indicate that the dPER-SLIMB interaction is highly dependent on phosphorylation of S47 and stimulated by other determinants such as S48 and additional phosphorylation events within the first 100 amino acids of dPER, most likely including S44 and/ or S45. As discussed below, mass spectrometry identified numerous phosphorylated residues on dPER and on going efforts are aimed at determining the contributions of all these phospho-residues to dPER stability. Based on the central importance of S47 in regulating dPER abundance and binding to SLIMB in our S2 cell culture system, herein we mainly focused on the physiological role of this site in the clockworks.

dPER S47 plays a key role in setting the pace of the clock

To investigate the physiological significance of our findings in whole animal systems, we generated transgenic flies that produce tagged versions of the $\operatorname{dPER}(\Delta 2-100)$, dPER(S44-48A), dPER(S47A), and dPER(S47D) proteins. The effects of the different transgenes were examined in the per-null wper ${ }^{\mathrm{O}}$ (Konopka and Benzer 1971), as well as wper $^{+}$genetic backgrounds. In addition, transgenic flies of the same genetic background but expressing a wildtype version of the dper transgene [herein referred to as $\mathrm{p}\{$ dper $(\mathrm{WT})\}]$ were included as controls (Kim et al. 2007; Ko et al. 2007). At least three independent lines of each genotype were analyzed. Flies were entrained for $4 \mathrm{~d}$ in 12 h:12 h LD (light/dark) cycle followed by $7 \mathrm{~d}$ in DD (constant dark) to determine their free-running period. As previously shown, wper $^{0}$ flies expressing the wild-type dper transgene exhibit strong rhythms with $\sim 24$-h periods (Table 1; Kim et al. 2007; Ko et al. 2007). Whereas $\mathrm{p}\{d$ per $(\Delta 2-100)\}$ and $\mathrm{p}\{d$ per $(\mathrm{S} 44-48 \mathrm{~A})\}$ transgenes failed to rescue the arrhythmic phenotype of the wper ${ }^{\mathrm{O}} \mathrm{mu}-$ tant, flies expressing $\mathrm{p}\{d \operatorname{per}(\mathrm{S} 47 \mathrm{~A})\}$ or $\mathrm{p}\{d \operatorname{per}(\mathrm{S} 47 \mathrm{D})\}$ transgenes showed high levels of rhythmicity but manifested long $(\sim 30.7 \mathrm{~h})$ and short $(-22.1 \mathrm{~h})$ periods, respectively (Table 1; Supplemental Fig. S3). This illustrates the physiological importance of S47 as a key phosphodeterminant in setting the pace of the Drosophila clock. In addition, the more severe behavioral phenotypes displayed by $\mathrm{p}\{d \operatorname{per}(\Delta 2-100)\}$ and $\mathrm{p}\{d \operatorname{dper}(\mathrm{S} 44-48 \mathrm{~A})\}$ flies compared with the single S47A mutant are consistent with results obtained in S2 cells (Figs. 1, 3), further supporting the idea that residues nearby to $\$ 47$ play supportive roles in regulating dPER metabolism.

The different transgenes manifested similar behavioral phenotypes in the per $^{+}$compared with those observed in the per $^{\mathrm{O}}$ genetic background, indicating they behave in a dominant manner (Table 1). The combined results indicate that when the stability of dPER increases, the period of the clock lengthens and at some threshold value further increases in dPER stability are incompatible with oscillator function leading to arrhythmia. Interestingly, although the period is longer in the per $^{\mathrm{O}}$ compared with per $^{+}$background for $\mathrm{p}\{d$ per(S47A) $\}$ flies, essentially identical periods were observed in either genetic background for flies expressing $\mathrm{p}\{d p e r(\mathrm{~S} 47 \mathrm{D})\}$. This suggests that despite the presence of wild-type dPER, most, if not all, the intracellular clocks are running at the accelerated pace set by dPER(S47D).

\section{dPER protein and $m R N A$ cycles reflect mutant circadian phenotypes}

To examine the dPER protein profiles of the mutants, ${ }_{\text {wper }}{ }^{\mathrm{O}}$ flies expressing wild-type or mutant dper transgenes were collected at different times throughout a daily cycle and head extracts probed by immunoblotting. As shown previously, dPER is first observed as a newly synthesized hypophosphorylated species (fastest migrating species) at around ZT8, undergoes progressive increases in phosphorylation, peaks in abundance at around ZT20 and attains the most highly phosphorylated isoforms around ZT4, concomitant with rapid decreases in levels (Fig. 4A; Edery et al. 1994). As expected based on the behavioral results, the $\mathrm{dPER}(\mathrm{S} 47 \mathrm{~A})$ and $\mathrm{dPER}(\mathrm{S} 47 \mathrm{D})$ proteins showed daily rhythms in abundance and phosphorylation, but with some significant differences as compared with $\operatorname{dPER}(\mathrm{WT})$ (Fig. 4A-C). In the case of the 
Table 1. Locomotor activity rhythms of dper mutants and control transgenic flies ${ }^{\mathrm{a}}$

\begin{tabular}{|c|c|c|c|c|}
\hline Genotype $^{\mathrm{b}}$ & Period $(\mathrm{h} \pm \mathrm{SEM})$ & Power ${ }^{\mathrm{c}}$ & Rhythmicity $(\%)^{\mathrm{d}}$ & Total flies ${ }^{\mathrm{e}}$ \\
\hline$w^{1118}$ & $23.5 \pm 0.08$ & 72.2 & 81.2 & 32 \\
\hline${ }{ }^{2} r^{0}$; per $^{+}$-HAHis (M16) & $23.3 \pm 0.04$ & 85.1 & 96.7 & 30 \\
\hline wper ${ }^{\circ}$ & $\mathrm{AR}^{\mathrm{f}}$ & 0 & 0 & 32 \\
\hline wper $^{0}$; per(S47A)-HAHis (M28) & $30.7 \pm 0.17$ & 63.9 & 83.9 & 31 \\
\hline wper $^{0} ;$ per(S47A)-HAHis (F15) & $30.7 \pm 0.1$ & 107.9 & 100 & 31 \\
\hline wper $^{\circ}$; per(S47D)-HAHis (M7) & $22.5 \pm 0.75$ & 105.2 & 93.5 & 31 \\
\hline wper $^{0} ;$ per(S47D)-HAHis (F31) & $22.1 \pm 0.07$ & 92.1 & 100 & 30 \\
\hline wper $^{0} ; \operatorname{per}(\mathrm{S} 44-48 \mathrm{~A})$-HAHis (F27) & $35.5 \pm 0$ & 21 & 3.1 & 32 \\
\hline wper ${ }^{\circ}$ per(S44-48A)-HAHis (M32) & AR & 0 & 0 & 31 \\
\hline wper $^{0} ; \operatorname{per}(\Delta 2-100)$-HAHis $(\mathrm{F} 4)$ & $\mathrm{AR}$ & 0 & 0 & 28 \\
\hline wper $^{0} ; \operatorname{per}(\Delta 2-100)$-HAHis (M34) & $\mathrm{AR}$ & 0 & 0 & 30 \\
\hline wper $^{+} ;$per(S47A)-HAHis (M28) & $27.7 \pm 0.14$ & 46.5 & 58.6 & 29 \\
\hline wper $^{+}$; per(S47A)-HAHis (F15) & $27.2 \pm 0.1$ & 89.7 & 86.2 & 29 \\
\hline wper $^{+} ;$per(S47D)-HAHis (M7) & $22.5 \pm 0.05$ & 69.1 & 69 & 29 \\
\hline wper $^{+} ;$per(S47D)-HAHis (F31) & $22.8 \pm 0.06$ & 72.1 & 80.6 & 31 \\
\hline wper $^{+}$; per $(\mathrm{S} 44-48 \mathrm{~A})-\mathrm{HAHis}(\mathrm{M} 13)$ & $27 \pm 0$ & 25.8 & 3.1 & 32 \\
\hline wper $^{+}$; per(S44-48A)-HAHis (M32) & $30.5 \pm 0$ & 55.4 & 3.4 & 29 \\
\hline wper $^{+} ; \operatorname{per}(\Delta 2-100)$-HAHis $(\mathrm{F} 4)$ & $\mathrm{AR}$ & 0 & 0 & 30 \\
\hline wper $^{+}$; per $(\Delta 2-100)$-HAHis (M34) & $\mathrm{AR}$ & 0 & 0 & 32 \\
\hline
\end{tabular}

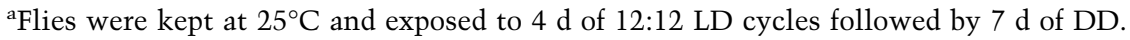

${ }^{b}$ Independent transgenic lines are designated by the numbers in parenthesis.

${ }^{c}$ Power is a measure of the strength or amplitude of the rhythm in arbitrary units.

${ }^{\mathrm{d}}$ Percentage of flies showing locomotor rhythms with a power value of $\geq 10$ and a width value of $\geq 2$.

${ }^{\mathrm{e}}$ Total number of flies that survived until the end of the experimental period.

${ }^{\mathrm{f}}(\mathrm{AR})$ Arrhythmic.

dPER(S47A) protein, hyperphosphorylated isoforms were found to persist throughout the daily cycle (Fig. 4B, open arrow). This is in sharp contrast to hyperphosphorylated isoforms of $\mathrm{dPER}(\mathrm{WT})$ proteins that undergo sharp decreases in abundance beginning in the early day (e.g., Fig. 4A,B, cf. ZT12). On the other hand, hyperphosphorylated isoforms of dPER(S47D) protein were never detected (Fig. 4C). Based on our results it is likely that hyperphosphorylated isoforms of dPER(S47D) are more readily degraded and never accumulate to detectable levels, although presently we cannot rule out the possibility that dPER(S47D) does not proceed through the entire hyperphosphorylation program in flies.

RNA levels of dper were attenuated in $\mathrm{p}\{\operatorname{dper}(\mathrm{S} 47 \mathrm{~A})\}$ flies but enhanced in p $\{$ dper(S47D)\} flies (Fig. 4G-H). A likely reason is that the more rapid clearance of $\mathrm{dPER}(\mathrm{S} 47 \mathrm{D})$ from the nucleus leads to decreased transcriptional repressor function and premature reactivation of dCLK-mediated transcription, whereas $\mathrm{dPER}(\mathrm{S} 47 \mathrm{~A})$ likely persists in the nucleus at elevated levels for longer, repressing dCLKmediated transcription and hence attenuating the amplitude of the dper RNA rhythm. Also, it is possible that phosphorylation might enhance the repressor ability of dPER (Nawathean and Rosbash 2004; Kim et al. 2007; Nawathean et al. 2007). Similar effects on the dper RNA abundance cycles were also observed when the mutants were analyzed in a per $^{+}$genetic background (Fig. 4G,H), consistent with the behavioral results (Table 1).

Neither dPER $(\Delta 2-100)$ nor dPER(S44-48A) proteins showed daily changes in abundance or phosphorylated state (Fig. 4D-F). The electrophoretic mobility of dPER(S44-48A) is higher than the most extensively phosphorylated iso- forms that we can detect for wild-type dPER (Fig. 4D, cf. lanes 5,6 and 1,2). Phosphatase treatment demonstrated that dPER(S44-48A) and wild-type dPER comigrate when dephosphorylated (data not shown), indicating that dPER(S44-48A) is very highly phosphorylated and the decreased mobility is not due to secondary issues, such as altered conformation. Although the electrophoretic mobility of $\operatorname{dPER}(\Delta 2-100)$ is faster because of the removal of 100 amino acids, it also is highly phosphorylated (data not shown). dper mRNA levels in p $\{$ dper $(\Delta 2-100)\}$ and $\mathrm{p}\{$ dper $(\mathrm{S} 44-48 \mathrm{~A})\}$ flies (in both wper ${ }^{0}$ and wper $^{+}$background) were essentially pegged at wild-type trough values, although some low amplitude cycling was observed for the dper(S44-48A) RNA, especially in a $p^{+}$background (Fig. 4I,J). Thus, in both S2 cells and flies, $\operatorname{dPER}(\Delta 2-100)$ and $\mathrm{dPER}(\mathrm{S} 44-48 \mathrm{~A})$ are very stable despite being highly phosphorylated, and exhibit more severe phenotypes compared with the singly S47A and S47D mutations. In addition, based on the RNA profiles we can infer that dPER $(\Delta 2-100)$ and $\operatorname{dPER}(S 44-48 \mathrm{~A})$ are effective in repressing dCLK-dependent transcriptional activation, consistent with prior work indicating that a more C-terminal region of dPER termed "CCID" (CLK-CYC inhibition domain) inhibits the trans-activation potential of dCLK (Chang and Reppert 2003).

GST-SLIMB pull-down assays using fly head extracts confirm results obtained in $S 2$ cells

To study SLIMB-dPER interactions in flies, head extracts were prepared from transgenic flies expressing 
Chiu et al.

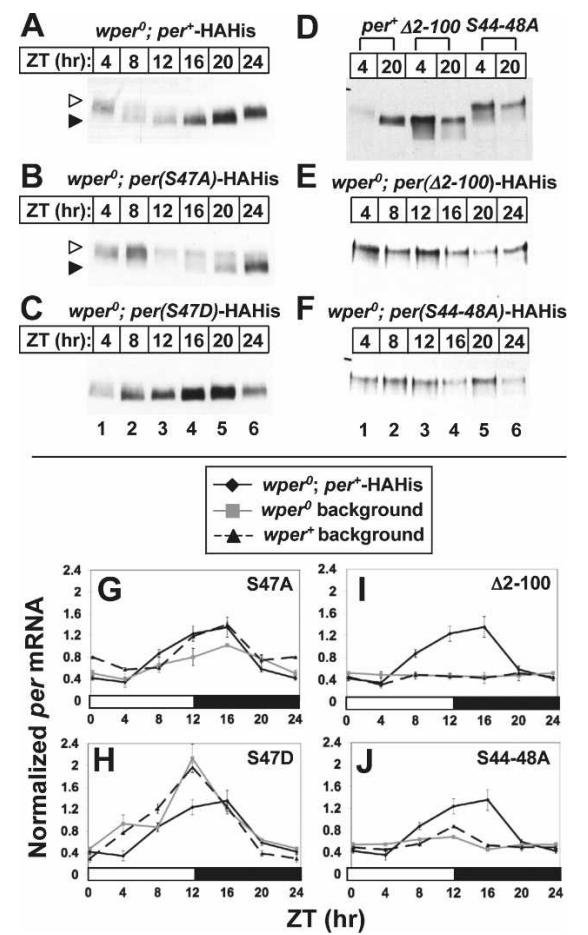

Figure 4. Daily cycles in dper protein and RNA are altered in dper mutant flies. Head extracts were prepared and used to detect either dPER protein $(A-F)$ or $d p e r$ RNA $(G-J)$. For the analysis of dPER protein, the different transgenes were evaluated in the wper $^{0}$ genetic background, whereas the levels of dper transcripts were determined in both wper ${ }^{0}$ and wper $^{+}$genetic backgrounds. The dPER protein profiles of wild type $(A, D$, lanes 1,2$)$ and four mutants $(B-F)$ were monitored throughout a daily cycle by immunoblotting in the presence of $\alpha$-HA. Open and closed triangles $(A, B)$ denote hyper- and hypophosphorylated dPER, respectively. $(G-J)$ dper mRNA profiles obtained by semiquantitative RT-PCR using $c b p 20$ for normalization. The control dper mRNA profile $(n=4)$ derived from wper $^{0}{ }^{0}$ per $^{+}$-HAHis flies is shown in all four plots to facilitate comparisons with each mutant profile $(n=2)$. The error bars shown are SEM.

wild-type or mutant variants of dPER in the wper ${ }^{0}$ background and incubated with GST-SLIMB resins (Fig. 5). In the case of $\mathrm{dPER}(\mathrm{WT})$ protein, binding to SLIMB is first detected at ZT20 with gradual increases that peak at around ZT4, despite the much lower overall levels of dPER at ZT4 (Fig. 5A [cf. lanes 1,2 and 5,6], B [cf. lanes 3-6 and 12-15]). The enhanced binding of $\operatorname{dPER}(\mathrm{WT})$ to SLIMB at ZT4 is consistent with the accelerated degradation of dPER during the early day. Likewise, binding of dPER(S44-48A) to SLIMB was highly inefficient at all times in a daily cycle (Fig. 5A, lanes 7,8), even though it is hyperphosphorylated and relatively abundant (Fig. 5A, lanes 3,4$)$. This further demonstrates that specific phospho-determinants within S44-48, rather than global hyperphosphorylation, plays a critical role in underlying highly efficient interactions between dPER and SLIMB. Nonetheless, although binding of $\operatorname{dPER}(\mathrm{S} 44-48 \mathrm{~A})$ is very low, it is above background levels observed with the control GST-resin (Fig. 5A, cf. lanes 7,8 to 11,12 ), suggesting that in the absence of phosphorylation at S47, phosphor- ylation of dPER at other sites can partly compensate and mediate weak interactions with SLIMB.

That phosphorylation at S47 and other regions contribute to the interaction between DPER and SLIMB is further supported by results obtained with the S47D and S47A variants. For example, although dPER(S47D) and dPER(WT) are both largely hypophosphorylated at ZT16, there is noticeable binding of dPER(S47D) to SLIMB, despite having substantially lower abundance compared with dPER(WT) (Fig. 5B, cf. lanes 1 and 4, and lanes 10 and 13). However, hyperphosphorylated isoforms of $\operatorname{dPER}(\mathrm{S} 47 \mathrm{D})$ exhibit relatively higher affinity for SLIMB compared with less grossly phosphorylated isoforms (Fig. 5B, cf. lanes 1,2 and 10,11). Significantly, although less extensively phosphorylated isoforms of $\mathrm{dPER}(\mathrm{WT})$ can interact with SLIMB (Fig. 5B, lanes 12,15), only the most highly phosphorylated isoforms of $\mathrm{dPER}(\mathrm{S} 47 \mathrm{~A})$ proteins are capable of binding to SLIMB (Fig. 5B, lane 17). In general, there is strong congruence with the results we obtained using dPER variants produced in S2 cells or fly extracts. Together, the findings indicate that the phosphorylated status of S47 is a critical but not absolute phospho-determinant modulating the affinity of SLIMB for dPER. Phosphorylation at other sites (including S44/45 and possibly other $\mathrm{S} / \mathrm{T}$ residues in the first 100 amino acids) can partially compensate for the absence of S47 phosphorylation and vice versa, but the presence of all the relevant phospho-determinants collaborate to generate a high-affinity binding site for SLIMB.

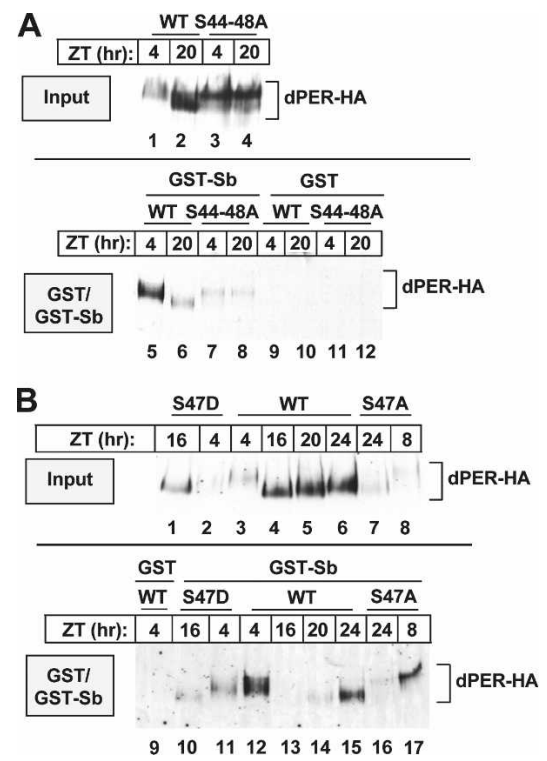

Figure 5. GST-SLIMB pull-down assays using fly head extracts further support S47 as a key phospho-determinant underlying SLIMB binding to dPER. $(A, B)$ Head extracts were prepared and aliquots containing equal amounts of total protein were incubated with glutathione resins bound with GST-SLIMB or GST. The relative amount of dPER-HA in the starting material (input) and that bound to the resins (GST/GST-Sb) was visualized by immunoblotting in the presence of $\alpha$-HA antibodies. 
Mass spectrometric analysis identifies multiple 'phospho-clusters' and suggests a molecular basis for the classic per' mutant

To better understand the dPER phosphorylation program we used a multiprotease approach in combination with mass spectrometry to comprehensively map dPER phosphorylation sites. This protocol was recently used to identify 21 serine/threonine phosphorylation sites on mouse PER2 (mPER2) (Schlosser et al. 2005; Vanselow et al. 2006). To identify DBT-dependent dPER phosphorylation sites, we established a stable S2 cell line that coexpressed Flagtagged dPER and untagged DBT under the inducible pMT promoter. A stable cell line that expressed dPER alone was also generated for the purpose of differentiating DBT-dependent phosphorylation sites from those targeted by endogenous kinases in S2 cells. Nine phosphorylated Ser or Thr residues on APER were identified in the absence of exogenously expressed DBT (Table 2). Among them, five are proline-mediated S/T sites, suggesting a role for CMGC kinases (Kannan and Neuwald 2004) in the Drosophila clock. Of note, S149, S151, and S153 are phosphorylated, consistent with prior work indicating that these sites are potential substrates of CKII and have a role in clock function (Lin et al. 2005). Eight of the nine sites targeted by endogenous kinases were also found to be phosphorylated in dPER purified from S2 cells coexpressing dPER and DBT. No phosphorylated tyrosine residues, either in the absence or presence of induced

Table 2. Mapping phosphorylation sites on dPER produced in Drosophila S2 cells

\begin{tabular}{ll}
\hline dPER $^{\mathrm{a}, \mathrm{b}}$ & \multicolumn{1}{c}{$\mathrm{dPER}+\mathrm{DBT}^{\mathrm{c}}$} \\
\hline- & {$[\mathrm{S} 40, \mathrm{~S} 42, \mathrm{~S} 44, \mathrm{~S} 45, \mathrm{~S} 47, \mathrm{~S} 48]^{\mathrm{d}, \mathrm{e}}$} \\
- & $\mathrm{S} 59, \mathrm{~S} 60$ \\
- & $\mathrm{S} 97$ \\
- & $\mathrm{S} 132$ \\
$\mathrm{~S} 149, \mathrm{~S} 151^{\mathrm{f}}, \mathrm{S} 153$ & $\mathrm{~S} 149, \mathrm{~S} 151, \mathrm{~S} 153$ \\
$\mathrm{~S} 596<\mathrm{P}>\mathrm{g}$ & $\mathrm{S} 585, \mathrm{~S} 589, \mathrm{~S} 596<\mathrm{P}>$ \\
$\mathrm{T} 610<\mathrm{P}>$ & $\mathrm{T} 610<\mathrm{P}>$ \\
$\mathrm{S} 661<\mathrm{P}>$ & $\mathrm{S} 661<\mathrm{P}>$ \\
- & $\mathrm{S} 773$ \\
- & $\mathrm{S} 826, \mathrm{~S} 828$ \\
$\mathrm{~T} 883<\mathrm{P}>$ & $\mathrm{S} 876, \mathrm{~T} 883<\mathrm{P}>, \mathrm{T} 889$ \\
$\mathrm{~S} 981<\mathrm{P}>,[\mathrm{T} 980, \mathrm{~T} 983, \mathrm{~S} 985]^{\mathrm{h}}$ & $\mathrm{T} 978<\mathrm{P}>, \mathrm{S} 981<\mathrm{P}>$ \\
- & $\mathrm{S} 1103$ \\
- & {$[\mathrm{S} 1129, \mathrm{~S} 1130<\mathrm{P}>]^{\mathrm{h}}$} \\
\hline
\end{tabular}

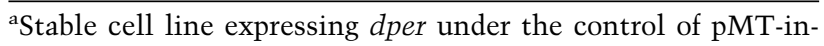
ducible promoter.

bdPER residues phosphorylated by endogenous kinase(s) in S2 cells.

${ }^{\mathrm{c} S t a b l e}$ cell line expressing dPER and DBT under the control of pMT-inducible promoter.

${ }^{\mathrm{d}}$ Two of the sites in the bracket are phosphorylated with S40 and S42 being less likely phosphorylated.

${ }^{\mathrm{e} A m i n o}$ acids are numbered according to sequence of dPER(amino acids 1-1224), GenBank accession number P07663. ${ }_{\mathrm{f}}^{\mathrm{f}} 151$ and S153 were identified previously as putative CKII phosphorylation sites (Lin et al. 2005).

$\mathrm{g}_{<\mathrm{P}>}$ denotes residues that are next to a proline.

${ }^{\mathrm{h}}$ Only one site in the bracket is phosphorylated. $d b t$, were detected, similar to mPER2 (Vanselow et al. 2006).

We identified an additional $16 \mathrm{~S} / \mathrm{T}$ residues in the presence of induced recombinant DBT, strong candidates for DBT-dependent phosphorylation sites. S47 was among peptide fragments that were phosphorylated, but unfortunately, we could not unequivocally determine the phosphorylated status of this residue. Nonetheless, mass spectrometry indicates that two out of the six Ser residues between S40 and S48 of dPER are phosphorylated in the presence of DBT (Table 2). This is consistent with the fact that in S2 cells and flies, dPER(S44-48A) exhibits a more severe phenotype compared with dPER(S47A). Ongoing work is aimed at evaluating contributions from other phospho-sites to dPER stability and binding to SLIMB.

Our mass spectral analysis also provides intriguing insights into the biochemical bases for several previously characterized per mutants (Supplemental Fig. S4). Most notably, the classic per $^{\text {Short=S }}$ mutation, which yields 19-h behavioral rhythms (Konopka and Benzer 1971), is an S589N mutation (Baylies et al. 1987; Yu et al. 1987). Phosphorylation of S589 was detected in the dPER + DBT sample but not dPER alone (Table 2). Finally, it is interesting to note that many of the phosphorylated residues on dPER are found in groups (Supplemental Fig. S4), suggesting distinct roles for the different phospho-clusters. Similar to mPER2 (Vanselow et al. 2006), most of the phosphorylation sites are concentrated in the central and Cterminal half of the protein, and no sites are found in the conserved PAS (Per-Arnt-Sim) domain. However, we did not detect specific phosphorylated residues that are conserved between dPER and mPER2, suggesting that although phosphorylation appears to regulate mammalian and insect PER proteins in similar manners, this might be attained by anchoring phospho-modules to conserved functional domains.

\section{Daily cycles in the phospho-occupancy of $S 47$}

To better evaluate the phosphorylated state of $S 47$, we generated phospho-specific polyclonal antibodies $(\alpha-\mathrm{pS} 47)$ (Fig. 6). The intensity of the immunoblotting signal obtained using $\alpha$-pS47 antibodies is greatly increased when dPER is coexpressed with DBT (Fig. 6A, lanes 1,2), consistent with the stimulatory effects of DBT on SLIMB binding to dPER. The specificity of our $\alpha$-pS47 antibodies was confirmed by demonstrating that the signal intensity is strongly attenuated by phosphatase treatment of dPER (Fig. 6A, lanes 2,3) and essentially abolished when probing the dPER(S47A) mutant (Fig. 6A, lanes 5,6). dPER containing phosphorylated S47 is detected as early as 12 $\mathrm{h}$ post- $d b t$ induction, but steadily increases in intensity peaking $\sim 10 \mathrm{~h}$ later, even though total dPER protein levels are lower at this later time point (Fig. 6B, cf. lanes 3,4 and 7,8$)$. The binding efficiency of dPER to SLIMB is roughly coincident with S47 phosphorylation (Fig. 6B), consistent with contributions from phosphorylation of S47, in addition to other sites.

It is noteworthy that there is relatively more phosphorylation of S47 at $22 \mathrm{~h}$ post- $d b t$ induction compared 


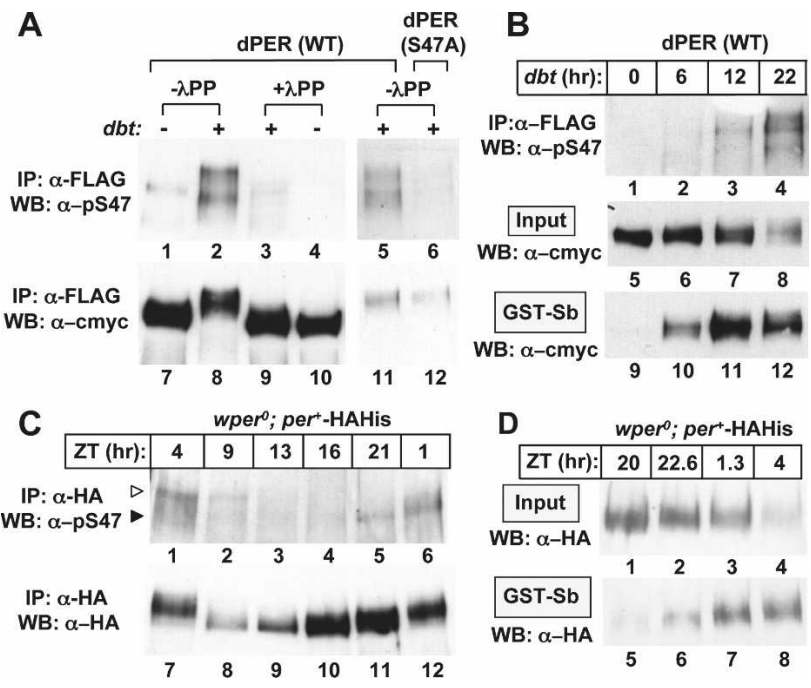

Figure 6. Phosphospecific antibodies detect pS47-containing isoforms of dPER in $\mathrm{S} 2$ cells and flies. (A) Extracts were prepared from S2 cells expressing either a wild-type (WT) or S47A version of pAc-3XFlag-His-dper/Tev100-6Xc-myc. In some cases cells were cotransfected with pMT- $d b t$-V5-His $(+)$, whereas this was omitted in other cases (-). dPER proteins were immunoprecipitated (IP) with $\alpha$-Flag beads, treated in the absence $(-\lambda \mathrm{PP})$ or presence $(+\lambda \mathrm{PP})$ of $\lambda$-phosphatase, and analyzed by Western blotting $(\mathrm{WB})$ in the presence of the indicated antibody (left of panel). (B, top) Cells were cotransfected with pAc-3XFlag-Hisdper-6Xc-myc and pMT-dbt-V5-His and collected at the indicated times post- $d b t$ induction. Extracts were prepared and divided into three separate aliquots: (1) One aliquot was immunoprecipitated (IP) with $\alpha$-Flag beads and analyzed by immunoblotting in the presence of $\alpha$-pS47 antibodies (top); (2) another aliquot was directly analyzed by immunoblotting in the presence of $\alpha$-c-myc antibodies (middle); and (3) a final aliquot was subjected to GST-SLIMB pull-down assays and bound material visualized by immunoblotting in the presence of $\alpha$-c-myc antibodies (bottom). $(C, D)$ Head extracts were prepared from $w_{p e r}^{0} ;$ per $^{+}$-HAHis flies collected at the indicated times $(\mathrm{ZT}) .(C)$ dPER-HAHis-containing immune complexes recovered using $\alpha$-HA beads were subjected to Western blotting (WB) in the presence of the indicated antibody (left of panel). Open and closed triangles indicate hyper- and hypophosphorylated pS47-containing isoforms of dPER, respectively. $(D)$ An aliquot was directly analyzed (top), whereas another fraction was subjected to GST-SLIMB pulldown assays and bound material analyzed (bottom).

with many hours earlier (Fig. 6B, lanes 3,4). This time frame is similar to that observed for the DBT-dependent global phosphorylation of full-length dPER (Fig. 1A) and more delayed compared with the conversion of the first 100-amino-acid fragment of dPER from hypo- to hyperphosphorylated isoforms (Fig. 2C). The later peak in S47 phospho-occupancy roughly coincides with rapid degradation in dPER levels, supporting the importance of this phospho-determinant in regulating dPER stability. It also suggests that DBT-mediated increases in the levels of S47 phosphorylation are relatively slow, gradually occurring over many hours, and that other phosphorylation events in the first 100 amino acids of dPER might undergo more rapid increases. Intriguingly, the majority of
dPER is stably bound to DBT within $5-10 \mathrm{~h}$ post- $d b t$ induction (Ko et al. 2002; data not shown), much before the peak in S47 phosphorylation. As such, the timing of when DBT binds dPER cannot account for the kinetics underlying the steady-state levels of phosphorylated S47. In this regard it is noteworthy that inhibiting protein PP1 and/or PP2A stimulates the hyperphosphorylation and/or degradation of dPER in cultured S2 cells as well as in flies (Sathyanarayanan et al. 2004; Fang et al. 2007). Indeed, the addition of okadaic acid, which at the concentration used here preferentially inhibits PP2A activity, is accompanied by temporal increases in the amount of dPER isoforms phosphorylated at S47 (Supplemental Fig. S5). Thus, it appears that PP2A (and/or PP1) stabilizes dPER by, at least in part, decreasing the phosphooccupancy of S47 and likely other sites. A reasonable model is that the balance between DBT and PP2A is a contributing factor in the slow hours-long kinetics underlying the ability of DBT to stimulate dPER binding to SLIMB and degradation.

To evaluate S47 phosphorylation within a physiological context we monitored its status throughout a daily cycle in flies using our $\alpha$-pS47 antibodies. Phosphorylation of S47 was first detected at around ZT21 (Fig. 6C, lane $5)$, soon after the time when $\mathrm{dPER}(\mathrm{WT})$ translocates to the nucleus (Shafer et al. 2002). This time frame coincides with the weak but initial detection of dPER-SLIMB interactions in flies (Fig. 6D), confirming that S47 phosphorylation and SLIMB binding are temporally aligned. pS47-containing dPER isoforms continue to accumulate throughout the late night and peak at around ZT4, when most dPER(WT) proteins are hyperphosphorylated and undergoing rapid degradation (Fig. 6C). A higher resolution time course showed that strong binding of dPER to SLIMB begins around ZT1 (Fig. 6D, lane 7) as dPER proteins that are phosphorylated at $\mathrm{S} 47$ are also undergoing global hyperphosphorylation (Fig. 6C, lanes 5,6). Prior work has shown that APER is already associated with DBT by ZT16 (Kloss et al. 2001; data not shown), indicating that steady-state increases in the phosphorylation of S47 do not occur quickly after DBT engages dPER. Thus, as observed in S2 cells, detectable phosphorylation of S47 begins at least several hours after DBT stably binds dPER and undergoes gradual increases in intensity that slightly precedes but is roughly coincident with global increases in dPER phosphorylation.

\section{Ser47 of Drosophila PER is phosphorylated by CKIঠ and DBT in vitro}

To determine whether DBT can directly phosphorylate S47, we expressed dPER(WT) tagged with Flag and c-myc epitopes in S2 cells, immunoprecipitated the recombinant $\mathrm{dPER}(\mathrm{WT})$ using $\alpha$-Flag resins, and subjected the immune complexes to in vitro kinase assays using commercially available CKI $\delta$ as well as recombinant DBT purified from S2 cells. The extent of S47 phosphorylation was evaluated using $\alpha$-pS47 antibodies. Both CKI $\delta$ and DBT triggered S47 phosphorylation (Fig. 7A [lanes 2,3], B [lane 4]), although phosphorylation by CKI $\delta$ appeared to 

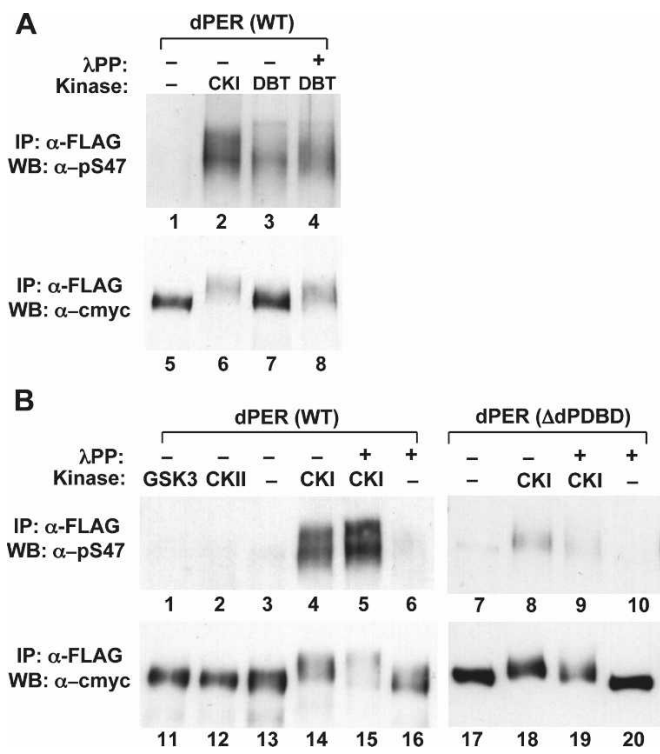

Figure 7. Ser47 of Drosophila PER is phosphorylated by DBT and CKI $\delta$ in vitro. $(A, B)$ Extracts were prepared from S2 cells express-ing wild-type or $\triangle$ dPDBD versions of pAc-3XFlag-Hisdper/Tev100-6Xc-myc. (Left) dPER proteins were immunoprecipitated using $\alpha$-Flag beads and subjected to in vitro kinase assays, followed by Western blotting (WB) in the presence of the indicated antibody. dPER containing immune complexes were either mock-treated $(-)$ or pretreated with $\lambda$-phosphatase $(+)$ prior to in vitro incubation in the absence $(-)$ or presence of the indicated kinase.

be more efficient and the majority of dPER was hyperphosphorylated as readily observed from the slower electrophoretic mobility (Fig. 7A, lanes 6,7). Phosphatase treatment of dPER did not abrogate the ability of DBT or CKIS to phosphorylate S47, strongly suggesting that priming by other kinases is not a prerequisite for S47 phosphorylation (Fig. 7A,B). To the contrary, phosphatase-treated dPER was more efficiently phosphorylated in our in vitro assays using DBT or CKIס, perhaps because some dPER sites phosphorylated by endogenous kinases in S2 cells antagonize DBT/CKI $\delta$ kinase activity on S47. Other clock relevant kinases such as GSK3 $\beta /$ SHAGGY and CKII (Martinek et al. 2001; Lin et al. 2002; Akten et al. 2003) failed to stimulate S47 phosphorylation (Fig. 7B, lanes 1,2$)$. Although we cannot rule out that priming at other sites by DBT is required for S47 phosphorylation, we tested a number of dPER variants with mutated Ser/ Thr residues in the first 100 amino acids and the efficiency of in vitro phosphorylation of S47 by DBT was similar or better compared with the control situation (data not shown).

\section{Phosphorylation at Ser47 requires the DBT-binding domain of Drosophila PER}

Prior mutational analysis showed that the major DBT docking site on dPER (dPDBD) does not contribute to DBT-dependent hyperphosphorylation by being a region that is extensively phosphorylated (Kim et al. 2007), confirmed here by mass spectrometry (Table 2). Rather, it appears that the main role of the $\mathrm{APDBD}$ in regulating dPER phosphorylation and stability is to serve as a docking site that enables stable interactions with DBT and possibly CKII or other kinases (Kim et al. 2007; Nawathean et al. 2007). To explore the relationship of the dPDBD to S47 phosphorylation we analyzed the ability of DBT/CKIo to phosphorylate the mutant dPER(AdPDBD) protein, using our in vitro kinase assay and extracts prepared from our previously characterized transgenic flies expressing dPER( $\triangle$ dPDBD) (Kim et al. 2007). Phosphorylation of S47 was greatly attenuated in the mutant version of dPER (Fig. 7B, cf. lanes 8,9 and 4,5). In addition, we did not detect phosphorylation of S47 for the dPER$(\triangle \mathrm{dPDBD})$ protein expressed in flies (Supplemental Fig. S6A), and it exhibits severely impaired ability to interact with SLIMB despite its high levels (Supplemental Fig. S6B, lanes 7,12).

\section{Discussion}

To better understand the physiological role of phosphorylation in regulating PER stability we used D. melanogaster as a model system. Using a range of strategies, including mutational analysis, mass spectrometry and phospho-specific antibodies we identified S47 as a key phospho-determinant regulating the efficiency of SLIMB binding to dPER. By evaluating the behavior of dPER mutants whereby amino acid 47 is constitutively "nonphosphorylated" (S47A) or "phosphorylated" (S47D), we show that the phospho-occupancy of S47 is a key biochemical throttle adjusting the pace of the clock. However, phosphorylation of S47 occurs within an atypical SLIMB-binding site. Additional DBT-dependent phosphorylated residues, which likely include one or more nearby Ser residues at amino acid 44/45 and possibly others within the first 100 amino acids, collaborate with pS47 to generate a high-affinity SLIMB-binding region on dPER. As such, the affinity of SLIMB for dPER is proportional to the degree of phospho-occupancy within an extended phosphorylation network centered on S47 that as a unit yields a graded response in the affinity of SLIMBdPER interactions. Attaining a high proportion of dPER molecules that are phosphorylated at S47 and other key sites mediating SLIMB binding is progressive and occurs several hours after DBT stably interacts with dPER via the centrally located dPDBD, likely because DBT "activity" is counterbalanced by TIM and protein phosphatases. We propose that the relatively slow assembly of a high-affinity SLIMB-binding site on dPER is at least partly "designed" to extend the time that dPER acts as a transcriptional repressor, critical in generating transcriptional feedback loops with daily time frames. Finally, our mass spectrometry analysis identify "hot spots" for phosphorylation, indicates that the majority of dPER phosphorylation is unrelated to direct effects on stability and sheds new insights into the underpinnings of several previously characterized mutants, including the classic per $^{\mathrm{s}}$ allele. 
Temporal regulation of dPER degradation by the slow DBT-dependent phosphorylation of a noncanonical SLIMB-binding site

Early studies identified DpSG $\Phi \mathrm{X}_{1+\mathrm{n}} \mathrm{pS}$ (pS, phosphorylated Ser; $\Phi$, any hydrophobic amino acid; $\mathrm{X}$, any amino acid) as the consensus motif for recognition by the $\beta$-TrCP/SLIMB F-box protein (Fuchs et al. 2004). Phosphorylation at both sites in this six amino acid consensus generally leads to a high-affinity $\beta$-TrCP/SLIMB-binding site. Indeed, the three negatively charged residues (Asp/Glu and two phospho-S/T residues) are important binding contacts underlying $\beta$-TrCP/substrate interactions (Wu et al. 2003). Furthermore, it is thought that the presence of an Asp/Glu at position 2 of the canonical binding domain can circumvent the need for a phospho-S/T at that position, as is the case for WeelA (Watanabe et al. 2004). However, accumulating evidence indicates that $\beta$-TrCP-binding sites can deviate from this consensus (Supplemental Fig. S1). For example, recent work on the Ci/Gli family of transcription factors suggests a novel class of degenerate and weaker $\beta$-TrCP-binding sites that extend beyond the standard six-amino-acid binding motif, especially for those missing a Gly at the third position (Smelkinson et al. 2007). It was suggested that for these extended $\beta$ TrCP/SLIMB-binding motifs, significant contributions are made by the local presence of nonpolar residues, such as those found in the motifs for $\mathrm{Ci}, \mathrm{Gli}$, and WeelA (Supplemental Fig. S1). Additional phosphorylation events at nearby regions are also thought to enhance the inherently weak binding affinities of extended $\beta$-TrCP-binding sites, enabling a more graded response compared with the standard sequence (Smelkinson et al. 2007).

The major SLIMB-dependent phospho-degron we identified $\left[{ }^{44} p^{\star} \mathrm{Sp}^{\star}\right.$ SGpSSGYGG ${ }^{52}$; where $\mathrm{p}^{\star}=$ possible phosphorylation] seems to include signature elements found in both the standard and extended $\beta$-TrCP-binding motifs. A rather unique feature of the SLIMB-binding domain on dPER is that it includes two SSG repeats. We show that $\mathrm{S} 47$ is phosphorylated, and based on mutational analysis and mass spectrometry, it is almost certain that either S44 and/or S45 are phosphorylated. A physiological role for S45 is further indicated by the per ${ }^{\mathrm{SLIH}} \mathrm{mu}-$ tant (S45Y) (Supplemental Fig. S4) that exhibits long periods (Hamblen et al. 1998), which based on our findings is likely due to reduced dPER-SLIMB interactions. Although changing S48 to Ala phenocopied the S47A mutation, the S48D mutation did not enhance binding to SLIMB, as was the case for S47D. Together with our results showing that S48A did not modulate phosphorylation of S47 (data not shown), the data strongly suggest that S48 has a non-phosphorylation-dependent role as a crucial structural element. Mass spectrometry identified two phospho-residues in a dPER peptide from amino acids 40-48 (Table 2). Thus, there might only be two negatively charged residues in the major SLIMB-binding site on dPER. It is possible that the presence of a SSG tandem and a Tyr at position 50 can compensate for the lack of a third acidic residue normally found in $\beta$-TrCP-binding sites. It is also highly probable that other, yet to be iden- tified, DBT-dependent phosphorylation sites besides those within the atypical SLIMB-binding site identified here contribute to enhancing SLIMB-dPER interactions.

The presence of numerous suboptimal phospho-determinants is thought to generate a graded response in the binding efficiencies of F-box proteins to substrates (e.g., Nash et al. 2001). The general molecular framework is that progressive increases in the phospho-occupancy of multiple phosphorylated residues eventually reaches a threshold value that drives sufficient F-box protein/substrate interactions to yield desired outcomes. As such, regulating the kinetics of phosphorylation within the phospho-network mediating F-box recognition is a key determinant in the timing of substrate degradation. In the case of animal PER proteins, they undergo progressive increases in global phosphorylation that occur over an 10-h time frame, whereby highly phosphorylated isoforms are associated with a rapid decline in levels (Edery et al. 1994; Lee et al. 2001).

What accounts for the hours-long kinetics underlying the gradual increases in phosphorylation of S47 and likely many other DBT-dependent sites on dPER? Based on the in vitro ability of DBT to phosphorylate S47 despite phosphatase treatment of dPER (Fig. 7), we do not believe that hierarchical phosphorylation based on prior priming is a major component in regulating the timing of when S47 is phosphorylated in vivo. Rather, our findings strongly suggest that the gradual build-up in the phospho-occupancy of S47 and other sites is at least partly based on a dynamic balance between DBT-mediated phosphorylation and the opposing activities of TIM and protein phosphatases. In agreement, blocking phosphatase activity strongly enhanced the abundance of phosphorylated S47 (Supplemental Fig. S5). Recent evidence suggests that the ability of TIM to stabilize dPER might be by acting as a bridge that facilitates the targeting of protein phosphatase activity toward dPER (Fang et al. 2007). Indeed, it is likely that the strong protective function of TIM on dPER partially overrides the destabilizing effects of the S47D mutant as it attains peak levels comparable with those of wild-type dPER (Fig. 4). Following this line of reasoning, we suggest that a major reason for the advanced dper RNA and protein cycles in the S47D mutant is that as TIM levels decline in the late night the "released" dPER(S47D) protein is no longer protected (or less so) and undergoes accelerated nuclear clearance, leading to an earlier disengagement from transcriptional repression, which advances the subsequent dper RNA and protein cycles (Fig. 4). Likewise, while this manuscript was under review a recent report showed that the CKI $\varepsilon$ tau mutation, which shortens rhythms in mice, has an "asymmetrical" effect on PER protein stability, preferentially accelerating nuclear clearance and hence advancing the molecular oscillations underpinning the clockworks (Meng et al. 2008). Thus, although differential phosphorylation plays a major role in setting the intrinsic stabilites of PER proteins, additional variables, such as phase-specific protein-protein interactions, are critical in the "readout" from these phospho-signals.

Many of the DBT-dependent phosphorylation sites 
that we identified using mass spectrometry do not lie within optimal CKI sites, suggesting that inefficient phosphorylation by DBT might also contribute to the overall rate of progressive increases in APER phosphorylation. It is also possible that the strong binding of DBT to the centrally located $\mathrm{dPDBD}$, while increasing the local concentration of DBT, could function as a slow "time-release capsule" whereby the disengagement of DBT is first required prior to phosphorylation of dPER residues at more distantly located sites.

Although the phosphorylation requirements and in vivo significance of recently identified regions on MPER 1 and mPER2 that interact with $\beta$-TrCP are not known, it is likely to also be based on noncanonical $\beta$-TrCP-binding sites (Supplemental Fig. S1; Eide et al. 2005; Shirogane et al. 2005). In addition, hyperphosphorylation of mammalian PER proteins requires a centrally located CKI-binding site (Lee et al. 2004; Eide et al. 2005; Shirogane et al. 2005). Therefore, mammalian PER proteins, especially mPER 1 and mPER 2 , are likely to be targeted by $\beta$-TrCP to the $26 \mathrm{~S}$ proteasome in a manner similar to that described here for dPER. This type of mechanism might also apply to other clock proteins such as FREQUENCY (FRQ) in Neurospora that undergoes daily changes in phosphorylation and stability that are remarkably similar to those observed for PER proteins (Garceau et al. 1997). In addition, the phosphorylated state of FRQ is regulated by casein kinases, protein phosphatases, and the rapid degradation of highly phosphorylated isoforms is mediated by the F-box protein FWD1, a homolog of $\beta$-TrCP (Yang et al. 2004; He and Liu 2005; Huang et al. 2007).

A systematic screen for dPER phosphorylation sites using mass spectrometry identifies phospho-clusters and sets the stage for understanding some of the classic clock mutants

An interesting feature of the distribution in phosphorylation sites on dPER that we identified using mass spectrometry is that they seem to concentrate in clusters, suggesting the presence of "phospho-modules" with different functions. Most of these clusters appear anchored by proline-directed phosphorylation sites, which are phosphorylated by endogenous kinases expressed in S2 cells. Of note, one such cluster is located in the dPER "short domain" (T585-T610). Mutations in this region result in animals with short periods (Konopka and Benzer 1971; Baylies et al. 1992; Rutila et al. 1992; Konopka et al. 1994). In fact, the mutated residues of two classic per mutants that have short periods, per $^{S}$ (S589N, 19-h period) and per $^{T}$ (G593D, 16-h period), are right in the heart of this cluster. S589 is phosphorylated in a DBT-dependent manner; and G593, when mutated, may affect phosphorylation at nearby S589 and/or S596. Although the per $^{S}$ mutants was isolated more than 35 years ago, our results provide the first biochemical understanding for the short period phenotype, suggesting that phosphorylation events in the "short domain," some of which are DBTdependent, may collaboratively function to slow down the clock. It is now becoming apparent that phosphorylation at different sites on PER proteins can result in differential effects on the pace of the clockworks, whereby some lead to faster clocks while others slow it down (e.g., Vanselow et al. 2006). The presence of phosphorylated residues with opposing outcomes on the speed of the clock can explain why mutations in CKI $\varepsilon / \delta / D B T$ can yield a variety of period-altering phenotypes from short to long, despite the fact that overall enzymatic activity is generally reduced (Price et al. 1998; Suri et al. 2000; Vanselow et al. 2006; Muskus et al. 2007).

A rather unanticipated finding is that the majority of dPER phosphorylation is unrelated to direct effects on stability. This is supported by the lack of detectable SLIMB binding to a dPER fragment only missing the first 100 amino acids despite extensive phosphorylation as inferred from being the region underlying the majority of phosphorylation-dependent electrophoretic mobility shifts and confirmed by our mass spectrometric analysis (Table 2; Supplemental Fig. S4). Other lines of evidence also imply that a significant amount of multiphosphorylation is not linked to direct effects on PER stability. For example, abolishing phosphorylation at many centrally located sites on mPER3 does not attenuate CKI-mediated in vitro interactions with $\beta$ - TrCP (Shirogane et al. 2005). Also, a trans-dominant version of CKII reduced global hyperphosphorylation of dPER without major effects on its levels (Smith et al. 2008).

Thus, there are likely to be at least two functionally distinct DBT-dependent phosphorylation programs regulating different aspects of PER metabolism and activity: one that controls $\beta$-TrCP/SLIMB binding, and another that integrates with other kinases, such as CKII, to modulate nuclear entry/accumulation and/or ability to function as a transcriptional repressor. Indeed, our mass spectrometric analysis of dPER identified numerous phosphorylation sites in a putative nuclear localization site and within the CCID mediating dPER inhibition of CLK-mediated transcription (Supplemental Fig. S4; Chang and Reppert 2003). Variants of dPER missing the major DBT docking site are hypophosphorylated and weak repressors (Kim et al. 2007; Nawathean et al. 2007). However, the relationship between hyperphosphorylation and repressor potency is not clear, as the DBT docking site on dPER also functions as a molecular scaffold for DBT and perhaps CKII-mediated inhibition of CLK-dependent transcription (Nawathean and Rosbash 2004; Yu et al. 2006; Kim et al. 2007; Nawathean et al. 2007). Nonetheless, it is clear that the DBT docking site is a critical nexus for coordinating multiple phosphorylation programs. A challenge is to examine the functions of the newly identified phosphorylation sites and dissect the mechanisms by which they regulate dPER metabolism and activity.

\section{Materials and methods}

Plasmids for $S 2$ cell expression and site-directed mutagenesis

Plasmids expressing pAc-dper-V5-His and pMT-dbt-V5-His were described in Kim et al. (2007) and Ko et al. (2002), respec- 
tively. Procedures for generating pAc-3XFlag-His-dper-6Xc-myc and pAc-3XFlag-His-dper/Tev100-6Xc-myc are described in the Supplemental Material. QuikChange site-directed mutagenesis kit (Stratagene) was used to generate $d p e r$ mutations using the various vectors described above as templates (see the Supplemental Material for primer sequences). Procedures for generating pMT-3XFlag-His-dper, pMT- $d b t$, pMT-gst, and pMT-gstslimb plasmids are discussed in the Supplemental material.

\section{S2 cell culture and transfection}

S2 cells and DES expression medium were obtained from Invitrogen, and transient transfections were performed using Effectene (Qiagen) according to the manufacturer's instructions. For each transient transfection, $0.8 \mu \mathrm{g}$ of different $d p e r$ containing plasmids and $0.2 \mu \mathrm{g}$ of pMT- $d b t$-V5-His or empty control pMTV5-His plasmids were used. Expression of $d b t$ was induced by adding $500 \mu \mathrm{M} \mathrm{CuSO}_{4}$ to the culture media $36 \mathrm{~h}$ after transfection. Stable cell lines expressing pMT-3XFlag-His-dper alone or with pMT- $d b t$, pMT-3XFlag-His- $d b t$, pMT-gst, and pMT-gstslimb were generated using calcium phosphate transfection kit (Invitrogen), and induction of pMT-driven expression was achieved using $500 \mu \mathrm{M} \mathrm{CuSO}_{4}$. For experiments in which the proteasome inhibitor MG132 (50 $\mu \mathrm{M}$; Sigma) and cycloheximide $(10 \mu \mathrm{g} / \mathrm{mL}$; Sigma) were used, they were added $4 \mathrm{~h}$ prior to cell harvest. These drugs were not used in the time-course experiments shown.

\section{Phosphorylation site mapping}

Hygromycin-resistant stable cell lines expressing pMT-3XFlagHis-dper alone or with pMT- $d b t$ were established for dPER purification (see Supplemental Material for cell culture conditions and purification procedures). Phosphorylation site mapping using mass spectrometry was performed as described in Schlosser et al. (2005). Data analysis was essentially performed as described previously (Schlosser et al. 2007).

\section{Transgenic flies and locomotor activity assays}

To generate transgenic flies carrying $d p e r$ mutations, we used a previously characterized vector that contains a $13.2-\mathrm{kb} d p e r$ genomic fragment tagged with the HA epitope and multiple histidine residues (10XHis) at the C-terminal (13.2per -HAHis) $^{+}$ (Lee et al. 1998). A XbaI-BamHI subfragment of this vector (including sequences encoding amino acids 1-870 of dPER) was subcloned into pGEM7 vector (Promega). This was used as the template for site-mutagenesis using the QuikChange Mutagenesis Kit (Stratagene). See the Supplemental Material for primer sequences. Mutated dper regions were confirmed by DNA sequencing, and used to replace the corresponding fragment in the 13.2per ${ }^{+}$-HAHis plasmid. Injection of $w^{1118}$ embryos was performed by Genetic Services, Inc., and transgenic flies expressing dper (wild-type or mutant) transgenes in $w^{1118}$ per $^{+}$(referred to as wper $\left.^{+}\right)$and $w^{1118}$ per $^{0}\left(\right.$ wper $\left.{ }^{0}\right)$ backgrounds were generated as described in Kim et al. (2007). Flies were kept at $25^{\circ} \mathrm{C}$ and entrained for at least three cycles of 12-h light:12-h dark (12:12 LD, where zeitgeber time $[\mathrm{ZT}] 0$ is the start of the light period), followed by at least $7 \mathrm{~d}$ in constant dark conditions (DD) for determination of free-running period. The measurement of fly locomotor activity rhythms was as previously described (Kim et al. 2007). To evaluate fly materials for Western blotting, GSTSLIMB pull-down, or immunoprecipitation, we entrained flies at $25^{\circ} \mathrm{C}$ in $12: 12 \mathrm{LD}$ for $3 \mathrm{~d}$, and collected them at the indicated $\mathrm{ZT}$ on the fourth day.
Generation of 547 phosphospecific antibodies and immunobloting

Rabbits were immunized with a 15 -amino-acid peptide (amino acid 40-SGSHSSGPSSGYGGKP—amino acid 54; where $\mathrm{p}=$ phosphate) (Proteintech Group, Inc.). Immunoblotting analysis was performed as described previously (Lee et al. 1998) with modifications detailed in the Supplemental Material.

\section{Immunoprecipitation (IP) and phosphatase ( $\lambda$-PP) treatment}

IP and $\lambda$-PP treatment were performed as described (Lee et al. 1998) with modifications detailed in the Supplemental Material.

\section{GST pull-down assay and TEV enzyme cleavage}

S2 cells expressing pAc-3XFlag-His-dper/Tev100-6Xc-myc variants were expressed with or without pMT- $d b t$-V5-His. Cells were treated with MG132 $(50 \mu \mathrm{M})$ and cycloheximide $(10 \mu \mathrm{g} /$ $\mathrm{mL}$ ) at $12 \mathrm{~h}$ (Fig. 3B) or at $16 \mathrm{~h}$ post- $d b t$ induction (Fig. 3C,D), and harvested $4 \mathrm{~h}$ later. For the time course experiment (Fig. $3 \mathrm{~A})$, cells were harvested at the indicated times without drug treatment. dPER proteins were extracted using EB2 (see the Supplemental Material). TEV cleavage was performed overnight at $4^{\circ} \mathrm{C}$ according to the manufacturer's instructions (Invitrogen). Mock or TEV-treated extracts were incubated with glutathione beads (Pierce) bound with GST or GST-SLIMB (purified from stable cell lines) usually for $4 \mathrm{~h}$ (up to overnight) at $4^{\circ} \mathrm{C}$. Bound dPER proteins were eluted with SDS sample buffer and resolved in 6\% SDS-PAGE for dPER(1-1224) and dPER(101$1224)$, or $16 \%$ SDS-PAGE for dPER(1-100). To generate GST and GST-SLIMB proteins, expression was induced from stable cell lines for $36 \mathrm{~h}$, and cells were lysed in GST lysis buffer (see the Supplemental Material). The extracts were then incubated with glutathione beads for $4 \mathrm{~h}$ at $4^{\circ} \mathrm{C}$ to achieve binding. GST pulldown assays for fly extracts were performed as described for S2 cell extracts (see the Supplemental Material for extract preparation), and bound dPER was resolved using 6\% SDS-PAGE.

\section{In vitro kinase assay}

S2 cells were transiently transfected with pAc-3XFlag-His-dper/ Tev100-6Xc-myc wild-type or $\triangle \mathrm{dPDBD}$ variants. S2 cells were harvested around $48 \mathrm{~h}$ after transfection without any drug treatment and dPER proteins were extracted using EB2 (see the Supplemental Material). After IP (see the Supplemental Material) to obtain dPER proteins, samples were subjected to $\lambda$-PP or mock treatment. Samples were subsequently washed three times with appropriate reaction buffers (CKI, CKII, or GSK3; New England Biolabs). Kinase assays were performed for $30 \mathrm{~min}$ at $30^{\circ} \mathrm{C}$ using $500 \mathrm{U}$ of CKIס, CKII, GSK3 (New England Biolabs), or 0.5 $\mu \mathrm{g}$ of Flag-tagged DBT purified from a stable S2 cell line expressing pMT-3XFlag-His- $d b t$ (reaction were supplemented with 100 $\mu M$ ATP [New England Biolabs]). Purification procedures are detailed in the Supplemental Material. All kinases used for in vitro phosphorylation studies were active as assayed using control substrates (data not shown).

\section{$R T-P C R$}

The relative dper mRNA levels were measured by semiquantitative RT-PCR as described previously (Kim et al. 2007). dper mRNA levels were normalized against noncycling cbp20 (capbinding protein 20) mRNA.

\section{Acknowledgments}

We thank H. Krause (University of Toronto) for pMT-3XFLAGHis. This work was supported by NIH grants NS049862 and 
NS061952 to J.C. and NIH34958 to I.E. Work in A.K.'s laboratory was supported by the Deutsche Forschungsgemeinschaft and the 6th EU framework program EUCLOCK.

\section{References}

Akten, B., Jauch, E., Genova, G.K., Kim, E.Y., Edery, I., Raabe, T., and Jackson, F.R. 2003. A role for CK2 in the Drosophila circadian oscillator. Nat. Neurosci. 6: 251-257.

Bae, K. and Edery, I. 2006. Regulating a circadian clock's period, phase and amplitude by phosphorylation: Insights from Drosophila. J. Biochem. 140: 609-617.

Baylies, M.K., Bargiello, T.A., Jackson, F.R., and Young, M.W. 1987. Changes in abundance or structure of the per gene product can alter periodicity of the Drosophila clock. Nature 326: 390-392.

Baylies, M.K., Vosshall, L.B., Sehgal, A., and Young, M.W. 1992. New short period mutations of the Drosophila clock gene per. Neuron 9: 575-581.

Chang, D.C. and Reppert, S.M. 2003. A novel C-terminal domain of Drosophila PERIOD inhibits dCLOCK:CYCLE-mediated transcription. Curr. Biol. 13: 758-762.

Darlington, T.K., Wager-Smith, K., Ceriani, M.F., Staknis, D., Gekakis, N., Steeves, T.D., Weitz, C.J., Takahashi, J.S., and Kay, S.A. 1998. Closing the circadian loop: CLOCK-induced transcription of its own inhibitors per and tim. Science 280: 1599-1603.

Dunlap, J.C. 1999. Molecular bases for circadian clocks. Cell 96: 271-290.

Edery, I., Zwiebel, L.J., Dembinska, M.E., and Rosbash, M. 1994. Temporal phosphorylation of the Drosophila period protein. Proc. Natl. Acad. Sci. 91: 2260-2264.

Eide, E.J., Woolf, M.F., Kang, H., Woolf, P., Hurst, W., Camacho, F., Vielhaber, E.L., Giovanni, A., and Virshup, D.M. 2005. Control of mammalian circadian rhythm by CKI $\varepsilon$-regulated proteasome-mediated PER2 degradation. Mol. Cell. Biol. 25: 2795-2807.

Fang, Y., Sathyanarayanan, S., and Sehgal, A. 2007. Post-translational regulation of the Drosophila circadian clock requires protein phosphatase 1 (PP1). Genes \& Dev. 21: 1506-1518.

Fuchs, S.Y., Spiegelman, V.S., and Kumar, K.G. 2004. The many faces of $\beta$-TrCP E3 ubiquitin ligases: Reflections in the magic mirror of cancer. Oncogene 23: 2028-2036.

Gallego, M. and Virshup, D.M. 2007. Post-translational modifications regulate the ticking of the circadian clock. Nat. Rev. Mol. Cell Biol. 8: 139-148.

Garceau, N.Y., Liu, Y., Loros, J.J., and Dunlap, J.C. 1997. Alternative initiation of translation and time-specific phosphorylation yield multiple forms of the essential clock protein FREQUENCY. Cell 89: 469-476.

Grima, B., Lamouroux, A., Chelot, E., Papin, C., LimbourgBouchon, B., and Rouyer, F. 2002. The F-box protein slimb controls the levels of clock proteins period and timeless. Nature 420: 178-182.

Hamblen, M.J., White, N.E., Emery, P.T., Kaiser, K., and Hall, J.C. 1998. Molecular and behavioral analysis of four period mutants in Drosophila melanogaster encompassing extreme short, novel long, and unorthodox arrhythmic types. Genetics 149: $165-178$.

Hardin, P.E. 2005. The circadian timekeeping system of Drosophila. Curr. Biol. 15: R714-R722. doi: 10.1016/j.cub.200. 508.019.

He, Q. and Liu, Y. 2005. Degradation of the Neurospora circadian clock protein FREQUENCY through the ubiquitin-proteasome pathway. Biochem. Soc. Trans. 33: 953-956.
Ho, M.S., Ou, C., Chan, Y.R., Chien, C.T., and Pi, H. 2008. The utility F-box for protein destruction. Cell Mol. Life Sci. doi: 10.1007/S0008-008-7592-6.

Huang, G., Chen, S., Li, S., Cha, J., Long, C., Li, L., He, Q., and Liu, Y. 2007. Protein kinase A and casein kinases mediate sequential phosphorylation events in the circadian negative feedback loop. Genes \& Dev. 21: 3283-3295.

Kannan, N. and Neuwald, A.F. 2004. Evolutionary constraints associated with functional specificity of the CMGC protein kinases MAPK, CDK, GSK, SRPK, DYRK, and CK $2 \alpha$. Protein Sci. 13: 2059-2077.

Kim, E.Y. and Edery, I. 2006. Balance between DBT/CKI $\varepsilon$ kinase and protein phosphatase activities regulate phosphorylation and stability of Drosophila CLOCK protein. Proc. Natl. Acad. Sci. 103: 6178-6183.

Kim, E.Y., Ko, H.W., Yu, W., Hardin, P.E., and Edery, I. 2007. A DOUBLETIME kinase binding domain on the Drosophila PERIOD protein is essential for its hyperphosphorylation, transcriptional repression, and circadian clock function. Mol. Cell. Biol. 27: 5014-5028.

Kloss, B., Price, J.L., Saez, L., Blau, J., Rothenfluh, A., Wesley, C.S., and Young, M.W. 1998. The Drosophila clock gene double-time encodes a protein closely related to human casein kinase I $\varepsilon$. Cell 94: 97-107.

Kloss, B., Rothenfluh, A., Young, M.W., and Saez, L. 2001. Phosphorylation of Period is influenced by cycling physical associations of Double-time, Period, and Tmeless in the Drosophila clock. Neuron 30: 699-706.

Ko, H.W., Jiang, J., and Edery, I. 2002. Role for Slimb in the degradation of Drosophila Period protein phosphorylated by Doubletime. Nature 420: 673-678.

Ko, H.W., DiMassa, S., Kim, E.Y., Bae, K., and Edery, I. 2007. Cis-combination of the classic per(S) and $\operatorname{per}(L)$ mutations results in arrhythmic Drosophila with ectopic accumulation of hyperphosphorylated PERIOD protein. J. Biol. Rhythms 22: 488-501.

Konopka, R.J. and Benzer, S. 1971. Clock mutants of Drosophila melanogaster. Proc. Natl. Acad. Sci. 68: 2112-2116.

Konopka, R.J., Hamblen-Coyle, M.J., Jamison, C.F., and Hall, J.C. 1994. An ultrashort clock mutation at the period locus of Drosophila melanogaster that reveals some new features of the fly's circadian system. J. Biol. Rhythms 9: 189-216.

Lee, C., Bae, K., and Edery, I. 1998. The Drosophila CLOCK protein undergoes daily rhythms in abundance, phosphorylation, and interactions with the PER-TIM complex. Neuron 21: $857-867$.

Lee, C., Bae, K., and Edery, I. 1999. PER and TIM inhibit the DNA binding activity of a Drosophila CLOCK-CYC/dBMAL1 heterodimer without disrupting formation of the heterodimer: A basis for circadian transcription. Mol. Cell. Biol. 19: 53165325.

Lee, C., Etchegaray, J.P., Cagampang, F.R., Loudon, A.S., and Reppert, S.M. 2001. Posttranslational mechanisms regulate the mammalian circadian clock. Cell 107: 855-867.

Lee, C., Weaver, D.R., and Reppert, S.M. 2004. Direct association between mouse PERIOD and CKI $\varepsilon$ is critical for a functioning circadian clock. Mol. Cell. Biol. 24: 584-594.

Lin, J.M., Kilman, V.L., Keegan, K., Paddock, B., Emery-Le, M., Rosbash, M., and Allada, R. 2002. A role for casein kinase $2 \alpha$ in the Drosophila circadian clock. Nature 420: 816-820.

Lin, J.M., Schroeder, A., and Allada, R. 2005. In vivo circadian function of casein kinase 2 phosphorylation sites in Drosophila PERIOD. J. Neurosci. 25: 11175-11183.

Martinek, S., Inonog, S., Manoukian, A.S., and Young, M.W. 2001. A role for the segment polarity gene shaggy/GSK-3 in the Drosophila circadian clock. Cell 105: 769-779. 
Meng, Q.J., Logunova, L., Maywood, E.S., Gallego, M., Lebiecki, J., Brown, T.M., Sladek, M., Semikhodskii, A.S., Glossop, N.R., Piggins, H.D., et al. 2008. Setting clock speed in mammals: The CK1 $\varepsilon \tau$ mutation in mice accelerates circadian pacemakers by selectively destabilizing PERIOD proteins. Neuron 58: 78-88.

Merrow, M., Mazzotta, G., Chen, Z., and Roenneberg, T. 2006. The right place at the right time: Regulation of daily timing by phosphorylation. Genes \& Dev. 20: 2629-2633.

Meyer, P., Saez, L., and Young, M.W. 2006. PER-TIM interactions in living Drosophila cells: An interval timer for the circadian clock. Science 311: 226-229.

Muskus, M.J., Preuss, F., Fan, J.Y., Bjes, E.S., and Price, J.L. 2007. Drosophila DBT lacking protein kinase activity produces long-period and arrhythmic circadian behavioral and molecular rhythms. Mol. Cell. Biol. 27: 8049-8064.

Nash, P., Tang, X., Orlicky, S., Chen, Q., Gertler, F.B., Mendenhall, M.D., Sicheri, F., Pawson, T., and Tyers, M. 2001. Multisite phosphorylation of a CDK inhibitor sets a threshold for the onset of DNA replication. Nature 414: 514-521.

Nawathean, P. and Rosbash, M. 2004. The doubletime and CKII kinases collaborate to potentiate Drosophila PER transcriptional repressor activity. Mol. Cell 13: 213-223.

Nawathean, P., Stoleru, D., and Rosbash, M. 2007. A small conserved domain of Drosophila PERIOD is important for circadian phosphorylation, nuclear localization, and transcriptional repressor activity. Mol. Cell. Biol. 27: 5002-5013.

Price, J.L., Dembinska, M.E., Young, M.W., and Rosbash, M. 1995. Suppression of PERIOD protein abundance and circadian cycling by the Drosophila clock mutation timeless. EMBO J. 14: 4044-4049.

Price, J.L., Blau, J., Rothenfluh, A., Abodeely, M., Kloss, B., and Young, M.W. 1998. double-time is a novel Drosophila clock gene that regulates PERIOD protein accumulation. Cell 94: 83-95.

Roenneberg, T. and Merrow, M. 2005. Circadian clocks-The fall and rise of physiology. Nat. Rev. Mol. Cell Biol. 6: 965971.

Rothenfluh, A., Young, M.W., and Saez, L. 2000. A TIMELESSindependent function for PERIOD proteins in the Drosophila clock. Neuron 26: 505-514.

Rutila, J.E., Edery, I., Hall, J.C., and Rosbash, M. 1992. The analysis of new short-period circadian rhythm mutants suggests features of $D$. melanogaster period gene function. $J$. Neurogenet. 8: 101-113.

Sathyanarayanan, S., Zheng, X., Xiao, R., and Sehgal, A. 2004. Posttranslational regulation of Drosophila PERIOD protein by protein phosphatase 2A. Cell 116: 603-615.

Schlosser, A., Vanselow, J.T., and Kramer, A. 2005. Mapping of phosphorylation sites by a multi-protease approach with specific phosphopeptide enrichment and NanoLC-MS/MS analysis. Anal. Chem. 77: 5243-5250.

Schlosser, A., Vanselow, J.T., and Kramer, A. 2007. Comprehensive phosphorylation site analysis of individual phosphoproteins applying scoring schemes for MS/MS data. Anal. Chem. 79: 7439-7449.

Shafer, O.T., Rosbash, M., and Truman, J.W. 2002. Sequential nuclear accumulation of the clock proteins period and timeless in the pacemaker neurons of Drosophila melanogaster. J. Neurosci. 22: 5946-5954.

Shirogane, T., Jin, J., Ang, X.L., and Harper, J.W. 2005. SCF $\beta$ TRCP controls clock-dependent transcription via casein kinase 1-dependent degradation of the mammalian period-1 (Per1) protein. J. Biol. Chem. 280: 26863-26872.

Smelkinson, M.G., Zhou, Q., and Kalderon, D. 2007. Regulation of Ci-SCFSlimb binding, Ci proteolysis, and hedgehog path- way activity by Ci phosphorylation. Dev. Cell 13: 481-495. Smith, E.M., Lin, J.M., Meissner, R.A., and Allada, R. 2008. Dominant-negative CK2 $\alpha$ induces potent effects on circadian rhythmicity. PLoS Genet. 4: e12. doi: 10.1371/journal.pgen.0040012.

Suri, V., Hall, J.C., and Rosbash, M. 2000. Two novel doubletime mutants alter circadian properties and eliminate the delay between RNA and protein in Drosophila. J. Neurosci. 20: 7547-7555.

Toh, K.L., Jones, C.R., He, Y., Eide, E.J., Hinz, W.A., Virshup, D.M., Ptacek, L.J., and Fu, Y.H. 2001. An hPer2 phosphorylation site mutation in familial advanced sleep phase syndrome. Science 291: 1040-1043.

Vanselow, K., Vanselow, J.T., Westermark, P.O., Reischl, S., Maier, B., Korte, T., Herrmann, A., Herzel, H., Schlosser, A., and Kramer, A. 2006. Differential effects of PER2 phosphorylation: Molecular basis for the human familial advanced sleep phase syndrome (FASPS). Genes \& Dev. 20: 2660-2672.

Watanabe, N., Arai, H., Nishihara, Y., Taniguchi, M., Watanabe, N., Hunter, T., and Osada, H. 2004. M-phase kinases induce phospho-dependent ubiquitination of somatic Weel by SCF $\beta-T r C P$. Proc. Nat1. Acad. Sci. 101: 4419-4424.

Wu, G., Xu, G., Schulman, B.A., Jeffrey, P.D., Harper, J.W., and Pavletich, N.P. 2003. Structure of a $\beta$-TrCP1-Skp1- $\beta$-catenin complex: Destruction motif binding and lysine specificity of the SCF $(\beta-\operatorname{TrCP} 1)$ ubiquitin ligase. Mol. Cell 11: 1445-1456.

$\mathrm{Xu}$, Y., Padiath, Q.S., Shapiro, R.E., Jones, C.R., Wu, S.C., Saigoh, N., Saigoh, K., Ptacek, L.J., and Fu, Y.H. 2005. Functional consequences of a CKI $\delta$ mutation causing familial advanced sleep phase syndrome. Nature 434: 640-644.

$\mathrm{Xu}$, Y., Toh, K.L., Jones, C.R., Shin, J.Y., Fu, Y.H., and Ptacek, L.J. 2007. Modeling of a human circadian mutation yields insights into clock regulation by PER2. Cell 128: 59-70.

Yang, Y., He, Q., Cheng, P., Wrage, P., Yarden, O., and Liu, Y. 2004. Distinct roles for PP1 and PP2A in the Neurospora circadian clock. Genes \& Dev. 18: 255-260.

Yu, Q., Jacquier, A.C., Citri, Y., Hamblen, M., Hall, J.C., and Rosbash, M. 1987. Molecular mapping of point mutations in the period gene that stop or speed up biological clocks in Drosophila melanogaster. Proc. Natl. Acad. Sci. 84: 784788.

Yu, W., Zheng, H., Houl, J.H., Dauwalder, B., and Hardin, P.E. 2006. PER-dependent rhythms in CLK phosphorylation and E-box binding regulate circadian transcription. Genes \& Dev. 20: 723-733. 


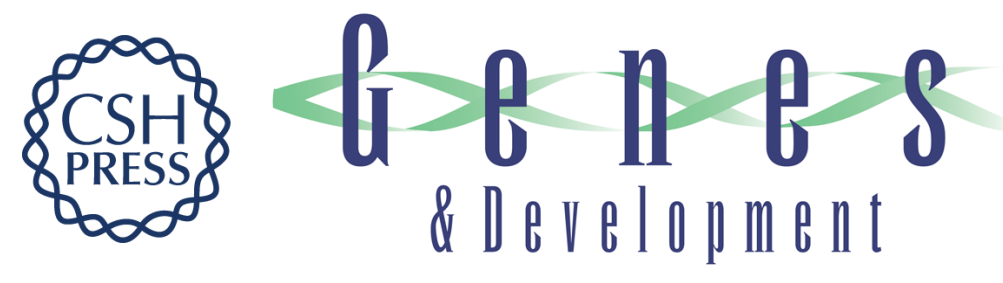

\section{The phospho-occupancy of an atypical SLIMB-binding site on PERIOD that is phosphorylated by DOUBLETIME controls the pace of the clock}

Joanna C. Chiu, Jens T. Vanselow, Achim Kramer, et al.

Genes Dev. 2008, 22:

Access the most recent version at doi:10.1101/gad.1682708

\section{Supplemental http://genesdev.cshlp.org/content/suppl/2008/07/01/22.13.1758.DC1 \\ Material \\ Related Content PERspective on PER phosphorylation \\ Justin Blau \\ Genes Dev. July , 2008 22: 1737-1740 \\ References This article cites 63 articles, 25 of which can be accessed free at: \\ http://genesdev.cshlp.org/content/22/13/1758.full.html\#ref-list-1 \\ Articles cited in: \\ http://genesdev.cshlp.org/content/22/13/1758.full.html\#related-urls \\ License \\ Email Alerting Receive free email alerts when new articles cite this article - sign up in the box at the top \\ Service right corner of the article or click here.}

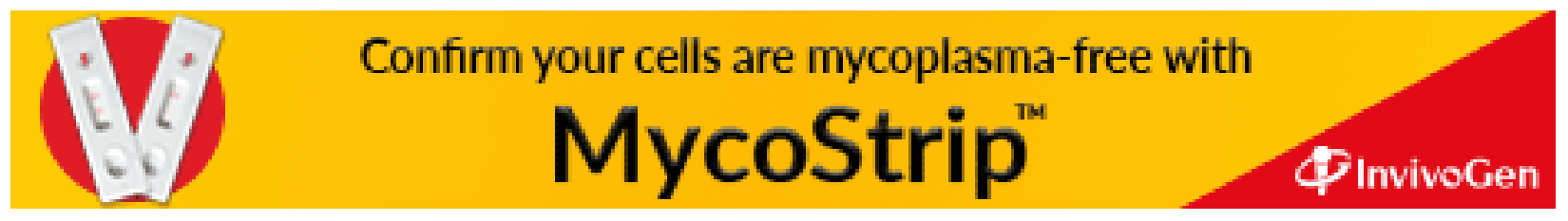

